\title{
Partially strong transparency conditions and a singular localization method in geometric optics
}

\author{
Yong $\mathrm{Lu}^{*}$ and Zhifei Zhang ${ }^{\dagger}$
}

\begin{abstract}
This paper focuses on the stability analysis of WKB approximate solutions in geometric optics with the absence of strong transparency conditions under the terminology of Joly, Métivier and Rauch. We introduce a compatible condition and a singular localization method which allows us to prove the stability of WKB solutions over long time intervals. This compatible condition is weaker than the strong transparency condition. The singular localization method allows us to do delicate analysis near resonances. As an application, we show the long time approximation of Klein-Gordon equations by Schrödinger equations in the non-relativistic limit regime.
\end{abstract}

\section{Keywords:}

Transparency condition, stability, WKB solution, singular localization method.

\section{Contents}

1 Introduction $\quad \mathbf{2}$

1.1 Setting and background ................... 3

1.2 Assumptions and main results . . . . . . . . . . . . . . 5

1.2.1 Smooth spectral decomposition ................... 5

1.2 .2 WKB solutions . . . . . . . . . . . . . . 5

1.2.3 Partially strong transparency . . . . . . . . . . . 8

1.2.4 Main result . . . . . . . . . . . . . . . . . 99 9

1.3 Structure of the paper . . . . . . . . . . . . . . 10

2 Transparency conditions and stabilities $\quad 10$

2.1 Weak transparency . . . . . . . . . . . . . . . . . . . . 10

2.2 Strong transparency and normal form method . . . . . . . . . . . . . 11

2.3 Absence of strong transparency . . . . . . . . . . . . . . . 13

* Mathematical Institute, Faculty of Mathematics and Physics, Charles University, Sokolovská 83, 18675 Praha, Czech Republic, luyong@karlin.mff.cuni.cz

${ }^{\dagger}$ School of Mathematical Science, Peking University, Beijing 100871, P. R. CHINA, zfzhang@math.pku.edu.cn 
4 Perturbed system and diagonalization $\quad 16$

4.1 Perturbed system near approximate solution . . . . . . . . . . . . 16

4.2 Diagonalization . . . . . . . . . . . . . . . . . . 17

5 Long time stability: Part I $\quad 19$

5.1 A singular localization . . . . . . . . . . . . . . . . . 19

5.2 Normal form reduction . . . . . . . . . . . . . . . . . . . . . . . . . . . . 22

5.3 End of the proof .......................... 24

6 Long time stability: Part II $\quad \mathbf{2 6}$

6.1 Refined singular localization . . . . . . . . . . . . . . . . 26

6.2 Refined normal form reduction . . . . . . . . . . . . . . . . 27

6.3 End of the proof $\ldots \ldots \ldots \ldots \ldots$

$\begin{array}{lll}7 & \text { Example and application } & 31\end{array}$

7.1 Setting and main result . . . . . . . . . . . . . . . . 32

7.2 Reformulation of the equation . . . . . . . . . . . . . . 32

7.3 Spectral decomposition . . . . . . . . . . . . . . . 33

7.4 WKB approximate solution ...................... 34

7.4 .1 WKB cascade . . . . . . . . . . . . . . 34

7.4 .2 WKB approximate solution . . . . . . . . . . . . 37

7.5 Partially strong transparency . . . . . . . . . . . . . . 38

7.6 Proof of Theorem $7.1 \ldots \ldots \ldots$. . . . . . . . . . . . . . 39

\section{Introduction}

In this paper, we consider the long time behavior of the solutions to Cauchy problems for symmetric hyperbolic systems of the following form

$$
\left\{\begin{array}{l}
\partial_{t} U+\frac{1}{\varepsilon} A\left(\partial_{x}\right) U+\frac{1}{\varepsilon^{2}} A_{0} U=B(U, U), \\
U(0, \cdot) \in H^{s}\left(\mathbb{R}^{d}\right)
\end{array}\right.
$$

where $U(t, x): \mathbb{R}_{+} \times \mathbb{R}^{d} \rightarrow \mathbb{R}^{N}$ is the unknown, $A\left(\partial_{x}\right)=\sum_{j=1}^{d} A_{j} \partial_{x_{j}}$ with $A_{j}, j=$ $1, \cdots, d$ real-valued symmetric matrices, $A_{0}$ is a real-valued skew-symmetric matrix and $B(\cdot, \cdot): \mathbb{R}^{N} \times \mathbb{R}^{N} \rightarrow \mathbb{R}^{N}$ is a symmetric bilinear application. The matrices $A_{j}$ are all of order $N \times N$. The initial datum $U(0)$ is supposed to be in Sobolev space $H^{s}$ with $s$ sufficiently large. 


\subsection{Setting and background}

We will consider solutions of (1.1) having the from

$$
U(t, x)=e^{-i \omega t / \varepsilon^{2}} U_{0,1}(t, x)+e^{i \omega t / \varepsilon^{2}} \bar{U}_{0,1}(t, x)+O(\varepsilon),
$$

which is highly oscillating in time with $\omega$ an appropriate characteristic temporal frequency satisfying

$$
\operatorname{det}\left(-i \omega+A_{0}\right)=0
$$

which is the so called dispersion relation; $\omega$ is also called the temporal wave number.

The study of highly oscillating solutions to hyperbolic systems falls in the framework of geometric optics. Considerable progress has recently been made in this field, especially following the works of Joly, Métivier and Rauch in the nineties (see for instance $[10,6,11,12,3]$, and [7] for an overview and references therein). In geometric optics, the main issue is the stability of a family of approximate solutions, namely WKB solutions, and the main obstacle is the resonance.

The hyperbolic system in (1.1) is symmetric semilinear. Then with $H^{s}, s>d / 2$ initial data, the local well-posedness is classical (see [20] or [22]). In spite of the presence of the large prefactors $1 / \varepsilon$ and $1 / \varepsilon^{2}$, the uniform $H^{s}$ estimate still holds due to the symmetry of $A_{j}$ and the skew-symmetry of $A_{0}$. Hence, with initial data that are uniformly bounded in $H^{s}, s>d / 2$, the classical existence time to Cauchy problem (1.1) is $O(1)$.

Our goal in this paper is to study the behavior of the solution to (1.1) beyond the classical time $O(1)$ up to long time of orders $O\left(1 / \varepsilon^{\gamma}\right)$ for some $\gamma>0$ given $O(1)$ initial data. This study falls in the framework beyond the weakly nonlinear regime of geometric optics, thus the classical results, for instance [10] - geometric optics for $O(1)$ amplitude, but $O(1)$ time, as well as [11] - diffractive optics for $O(1 / \varepsilon)$ time, but $O(\varepsilon)$ amplitude, do not apply. By assuming the global-in-time (or long time) existence of approximate solutions, we exhibit some sufficient conditions on (1.1), and introduce a singular localization method which allows us to make use of such sufficient conditions to show the existence as well as the stability of solutions over long time intervals. Such sufficient conditions are described in Section 1.2, in particular in the key Assumption 1.6. The singular localization method is introduced and described in Section 5 and Section 6.

As an application, we show in Section 7 that in the non-relativistic limit regime the quadratic Klein-Gordon equation can be well approximated by linear Schrödinger equations over long time intervals of order $O(1 / \varepsilon)$.

We point out that the condition imposed in the key Assumption 1.6 is analogous to, but weaker and more general than, the strong transparency condition exhibited by Joly, Métivier and Rauch in [12]. The strong transparency condition allows a control of the constructive interaction of characteristic waves at the resonances by a normal 
form reduction, thus leading to the stability of approximate WKB solutions. The transparency condition is analogous to the null conditions introduced by Klainerman in [13]; the normal form reduction allowed by the transparency property is analogous to the analysis of Shatah in [23]. As it will be shown in Section 7.5, the quadratic Klein-Gordon equation satisfies the condition imposed in Assumption 1.6 while it does not satisfy the strong transparency condition.

We also point out that the approximate linear transparency condition introduced in [5, Assumption 1.7], which is also weaker than the strong transparency condition, has similarities with our setting. To be precise, the condition in [5, Assumption 1.7] can be recovered by taking $\alpha=1 / 2$ in our Assumption 1.6. We remark that in our setting, $\varepsilon$ corresponds to $\sqrt{\varepsilon}$ in [5]. Moreover, the idea to decompose the integral form into two parts (see page 31 in [5]), where one part is the integral over a neighbourhood of resonances $D^{\varepsilon}:=\left\{\eta^{\prime} \in \mathbb{R}^{d}:\left|\psi^{\varepsilon}\left(\varepsilon \eta^{\prime}\right) \leq \sqrt{\varepsilon}\right|\right\}$ and the other part is the integral over the complement of $D^{\varepsilon}$, is essentially of the same sprit as our singular localization method. However, the analysis here is not simply a generalization of the argument in [5] from $\alpha=1 / 2$ to general $\alpha>0$. In particular, the singular localization method used in this paper can be employed to deal with the Klein-Gordon-wave equations (1.10) and (1.11) studied in [5] and to show the same stability results. But the analysis in [5] strongly relies on the typical structure of the system which we do not assume in this paper (see equation (1.9)). In particular, the block diagonal structure of the differential operator and the special coupling structure of the nonlinear terms play a crucial role for the stability analysis argument in $[5]$.

The strong transparency condition ensures the stability of WKB solutions. However, many (most) physical models in geometric optics do not fulfill the strong transparency condition, such as the Euler-Maxwell system, the Klein-Gordon system, the Maxwell-Landau-Lifshitz system, the Klein-Gordon-Zakhorov system, etc.. Thus, the study of the case where the strong transparency condition is not satisfied is highly important. In [18], Texier and the first author gave a systematic study concerning the case with the absence of the strong transparency condition. In particular, the article [18] contains a detailed account of how resonances may destabilize the WKB solutions. There was exhibited an almost sufficient and necessary condition for the stability of WKB solutions by giving a scalar index $\Gamma$ of which the positivity ensures instability and the negativity ensures stability.

However, the case $\Gamma=0$ is not included in the study of [18]. The case considered in this paper corresponds to a large family of subcases of the case $\Gamma=0$. Even if the scaling in this paper is different from the one in [18], the result obtained, as well as the method used in this paper could give some clear clues for the study in the scaling of [18] and others. 


\subsection{Assumptions and main results}

In this section, we state our main assumptions and results.

\subsubsection{Smooth spectral decomposition}

We first assume the symbol of the differential operator on the left-hand side of (1.1) admits a smooth spectral decomposition:

Assumption 1.1. We assume that the spectral decomposition

$$
A(\xi)+A_{0} / i=\sum_{j=1}^{J} \lambda_{j}(\xi) \Pi_{j}(\xi)
$$

is smooth, meaning that the eigenvalues $\lambda_{j}(\xi)$ and the eigenprojectors $\Pi_{j}(\xi)$ are smooth in $\xi \in \mathbb{R}^{d}$. Moreover, for any $1 \leq j \leq J$, we suppose that $\lambda_{j}(\cdot)$ and $\Pi_{j}(\cdot)$ are in the classical symbol class $S^{1}$ and $S^{0}$, respectively.

The definition of the symbol classes $S^{m}$ is classical and will be recalled in Section 3 .

\subsubsection{WKB solutions}

By WKB (approximate) solutions of (1.1) we mean truncated power series in $\varepsilon$, where each term in the series is a trigonometric polynomial in $\theta:=-\omega t / \varepsilon^{2}$, that approximately solves (1.1). Precisely, a WKB solution $U_{a}$ has the form

$U_{a}(t, x)=\sum_{n=0}^{K_{a}+1} \varepsilon^{n} \mathbf{U}_{n}(t, x, \theta), \quad \mathbf{U}_{n}(t, x, \theta)=\sum_{p \in \mathcal{H}_{n}} e^{i p \theta} U_{n, p}(t, x), K_{a} \in \mathbb{Z}_{+}, \mathcal{H}_{n} \subset \mathbb{Z}$,

which solves

$$
\left\{\begin{array}{l}
\partial_{t} U_{a}+\frac{1}{\varepsilon} A\left(\partial_{x}\right) U_{a}+\frac{1}{\varepsilon^{2}} A_{0} U_{a}=B\left(U_{a}, U_{a}\right)-\varepsilon^{K_{a}} R^{\varepsilon} \\
U_{a}(0, x)=U(0, x)-\varepsilon^{K} \psi^{\varepsilon}(x)
\end{array}\right.
$$

with $\left(R^{\varepsilon}, \psi^{\varepsilon}\right)$ bounded uniformly in $\varepsilon$ in some Sobolev spaces. Parameters $K_{a}$ and $K$ describe the level of precision of the WKB solution $U_{a}$. Here $\mathcal{H}_{n}$ are the harmonics sets. In particular, in this paper, the leading harmonics set is defined as

$$
\mathcal{H}_{0}:=\{-1,1\} \subset \mathcal{R}:=\left\{p: \operatorname{det}\left(-i p \omega+A_{0}\right)=0\right\} .
$$

The idea to find or construct such a WKB solution is quite straightforward, that is to plug a solution $U_{a}$ of the form (1.4) into the system (1.1), and then consider the equations at each order $\varepsilon^{n}, n=-2,-1, \cdots$. If one can solve the equations of 
order $\varepsilon^{n}$ up to some positive order $N_{a}$, then one can solve the original system (1.1) approximately, up to a small remainder of order $O\left(\varepsilon^{N_{a}+1}\right)$.

In this paper, we assume that there exits a global-in-time approximate solution for (1.1).

Assumption 1.2. Let $s>d / 2$. We assume the vector space $\operatorname{ker}\left(-i \omega+A_{0}\right)$ is of dimension one with $e_{1}$ a generator of norm one. We assume there exists $U_{a} \in$ $C_{b}\left([0, \infty) ; H^{s+1}\right) \cap C_{b}^{1}\left([0, \infty) ; H^{s}\right)$ solving $(1.5)$ for all $(t, x) \in(0, \infty) \times \mathbb{R}^{d}$ with $K_{a}=2, K=1$, and there holds the estimate

$$
\sup _{0<\varepsilon<1}\left(\left\|R^{\varepsilon}\right\|_{L^{\infty}\left(0, \infty ; H^{s}\right)}+\left\|\psi^{\varepsilon}\right\|_{H^{s}}\right)<+\infty .
$$

Moreover, $U_{a}$ is of the form (1.4) with $U_{n} \in C_{b}\left([0, \infty) ; H^{s+1}\right) \cap C_{b}^{1}\left([0, \infty) ; H^{s}\right), 0 \leq$ $n \leq K_{a}+1=3$; in particular, the leading term $U_{0}$ is of the form

$$
U_{0}=e^{-i \omega t / \varepsilon^{2}} U_{0,1}+e^{i \omega t / \varepsilon^{2}} U_{0,-1}
$$

where

$$
U_{0,1}(t, x)=g_{1}(t, x) e_{1}, \quad U_{0,-1}(t, x)=g_{-1}(t, x) e_{-1}, \quad g_{-1}:=\bar{g}_{1}, e_{-1}=\bar{e}_{1}
$$

for some scalar function $g_{1} \in C_{b}\left([0, \infty) ; H^{s+1}\right) \cap C_{b}^{1}\left([0, \infty) ; H^{s}\right)$.

The notation $\bar{a}$ stands for the complex conjugate of $a$.

Remark 1.3. To obtain our main result Theorem 1.8, the existence time and uniform bound for $U_{a}$ in Assumption 1.2 can be generalized to $U_{a} \in C_{b}\left(\left[0, \frac{T}{\varepsilon}\right] ; H^{s+1}\right) \cap$ $C_{b}^{1}\left(\left[0, \frac{T}{\varepsilon}\right] ; H^{s}\right)$ satisfying the uniform estimate

$$
\left\|U_{n}\right\|_{L^{\infty}\left(\left[0, \frac{T}{\varepsilon}\right] ; H^{s+1}\right)}+\left\|\partial_{t} U_{n}\right\|_{L^{\infty}\left(\left[0, \frac{T}{\varepsilon}\right] ; H^{s}\right)} \leq C<\infty
$$

for some constant $C$ independent of $\varepsilon$ and some time $T>0$ independent of $\varepsilon$.

The local-in-time WKB solutions to (1.1) can be constructed by using standard WKB expansion under the constrain (1.9) given later on. The main point of Assumption 1.2 is the global-in-time (or long time) existence and global-in-time (or long time) uniform bounds for the approximate solutions.

In the sequel of this section, we impose some compatibility conditions which ensure the existence of global-in-time approximate solutions such that Assumption 1.2 is satisfied.

Condition 1: The leading terms of the initial data satisfy:

$$
U(0)=U_{0,1}(0, x)+\bar{U}_{0,1}(0, x)+O(\varepsilon) \text { in } H^{s}, \quad U_{0,1}(0, x) \in \operatorname{ker}\left(-i \omega+A_{0}\right) .
$$

This is often called the polarization condition. 
Let $\pi_{p}$ be the orthogonal projection onto $\operatorname{ker}\left(-i p \omega+A_{0}\right)$ and $L_{p}^{-1}$ be the (partial) inverse of $L_{p}:=\left(-i p \omega+A_{0}\right)$ such that

$$
\pi_{p} L_{p}^{-1}=L_{p}^{-1} \pi_{p}=0, \quad L_{p} L_{p}^{-1}=L_{p}^{-1} L_{p}=\mathrm{Id}-\pi_{p} .
$$

Condition 2: We suppose for any $p \in \mathbb{Z}$ and any $\xi \in \mathbb{R}^{d}$ there holds

$$
\pi_{p} A(\xi) \pi_{p}=0
$$

Condition 3: We suppose for any $p \in \mathbb{Z}$ there holds

$$
\pi_{p} \sum_{p_{1}+p_{2}=p} B\left(\pi_{p_{1}}, \pi_{p_{2}}\right)=0
$$

Condition 4: We suppose furthermore for any $p \in \mathbb{Z}$ and any $\xi \in \mathbb{R}^{d}$ that

$$
\begin{aligned}
& \pi_{p} A(\xi) L_{p}^{-1} A(\xi) L_{p}^{-1} A(\xi) \pi_{p}=0, \\
& \pi_{p} A(\xi) L_{p}^{-1} \sum_{p_{1}+p_{2}=p} B\left(\pi_{p_{1}}, \pi_{p_{2}}\right)+2 \pi_{p} \sum_{p_{1}+p_{2}=p} B\left(\pi_{p_{1}}, L_{p_{2}}^{-1} A(\xi) \pi_{p_{2}}\right)=0 .
\end{aligned}
$$

We then have

Proposition 1.4. Assumption 1.2 holds true if Condition 1, Condition 2, Condition 3 and Condition 4 are all satisfied. More precisely, we have

(i). Under Condition 1 and Condition 2 and the additional assumption:

$$
\operatorname{ker}\left(-i p \omega+A_{0}\right)=\{0\} \text { for any } p \text { satisfying }|p| \geq 2 ; \quad \pi_{0} B\left(\pi_{1}, \pi_{-1}\right)=0,
$$

one can construct a uniformly bounded local-in-time WKB solution $U_{a}$ solving (1.5) with arbitrary $K_{a}$ and $K$.

(ii). Under Condition 1, Condition $\mathbf{2}$ and Condition 3, one can construct a uniformly bounded global-in-time WKB solution $U_{a}$ solving (1.5) with $K_{a}=$ $K=1$.

(iii). Under Condition 1, Condition 2, Condition 3 and Condition 4, one can construct a uniformly bounded global-in-time WKB solution $U_{a}$ solving (1.5) with $K_{a}=2, K=1$.

The proof of Proposition 1.4 can be done by employing the standard WKB expansion for which we give a detailed description in Section 7.4. The WKB expansion in Section 7.4 is done for a specific example instead of the general case, but the procedure is essentially the same. So here we omit the proof of Proposition 1.4. We point out that in statements (ii) and (iii) in Proposition 1.4, we do not 
need to assume the additional assumption (1.11) to make sure the leading term of the approximate solution has the form (1.7). Indeed, Condition 3, together with Condition 1, allows us to choose trivial solutions $U_{0, p} \equiv 0$ for any $p \notin\{-1,1\}$ in the WKB expansion.

Finally we give a remark concerning the conditions exhibited above.

Remark 1.5. Concerning condition (1.9), it was shown in [15, 6] (see also [24, Proposition 2.6] a unified proof for such algebraic lemmas) that for any $\xi \in \mathbb{R}^{d}$, there holds

$$
\pi_{p} A(\xi) \pi_{p}=\nabla_{\xi} \lambda_{j_{p}}(0) \cdot \xi
$$

where $\lambda_{j_{p}}$ is the eigenmode in Assumption 1.1 such that $\lambda_{j_{p}}(0)=-p \omega$. Thus, condition (1.9) means $\nabla_{\xi} \lambda_{j_{p}}(0)=0$. This associates with the condition in Assumption 1.5 in [5] saying that $(-p \omega, 0)$ is a local extremum of every branch of the characteristic defined in (2.1).

The condition in (1.10) corresponds exactly to the weak transparency condition introduced by Joly, Métivier and Rauch in [12]. See also (2.2) for the precise description.

\subsubsection{Partially strong transparency}

Now we give our key assumption:

Assumption 1.6. For any $p \in\{-1,1\}$ and any $1 \leq j, j^{\prime} \leq J$, there exists some constant $C$ and $0<\alpha_{j, j^{\prime}, p} \leq 1$ such that

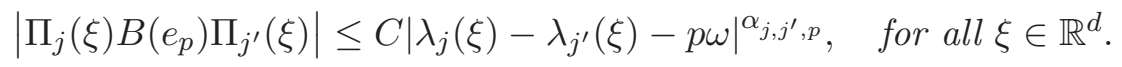

The vectors $e_{1}$ and $e_{-1}$ are introduced in Assumption 1.2. The linear operator $B\left(e_{p}\right)$ is defined as $B\left(e_{p}\right) V:=B\left(e_{p}, V\right)$ for any $V \in \mathbb{C}^{N}$.

Moreover, for any $p \in\{-1,1\}$ and any $1 \leq j, j^{\prime} \leq J$, the resonance set

$$
R_{j, j^{\prime}, p}:=\left\{\xi \in \mathbb{R}^{d}: \lambda_{j}(\xi)-\lambda_{j^{\prime}}(\xi)-p \omega=0\right\}
$$

is compact. If $R_{j, j^{\prime}, p}=\emptyset$, there exits $c_{j, j^{\prime}, p}>0$ such that

$$
\left|\lambda_{j}(\xi)-\lambda_{j^{\prime}}(\xi)-p \omega\right| \geq c_{j, j, p} \quad \text { for all } \xi \in \mathbb{R}^{d} .
$$

Given Assumption 1.6, we further define the exponent:

$$
\alpha:=\min _{j, j^{\prime}, p} \alpha_{j, j^{\prime}, p}
$$

If $\alpha=1$, Assumption 1.6 becomes the strong transparency assumption (see [12] and Section 2.2 later on). If $\alpha=1 / 2$, Assumption 1.6 implies [5, Assumption 
1.7]. Because of the presence of the fractional power $0<\alpha \leq 1$, we may call such condition imposed in Assumption 1.6 as the partially strong transparency condition.

We will show that under Assumption 1.6, the approximate solution assumed in Assumption 1.2 is stable up to time of order $O\left(1 / \varepsilon^{\alpha}\right)$. Now we give an additional assumption, which allows us to show the stability up to even longer time $t_{\varepsilon}$ which is of order

$$
t_{\varepsilon}=O\left(1 / \varepsilon^{2 \alpha}\right), \text { if } \alpha \leq 1 / 2 ; \quad t_{\varepsilon}=O(1 / \varepsilon), \text { if } \alpha \geq 1 / 2 .
$$

Assumption 1.7. If for some $\left(j, j^{\prime}, p\right)$, the component $\alpha_{j, j^{\prime}, p}$ in (1.13) cannot be chosen equal to 1 , we assume that either $\lambda_{j}$ or $\lambda_{j^{\prime}}$ is identically a constant.

The case $\alpha_{j, j^{\prime}, p}<1$ corresponds to the case where the interaction coefficient $\Pi_{j}(\xi) B\left(e_{p}\right) \Pi_{j^{\prime}}(\xi)$ is not strongly transparent. The nontransparent interaction coefficients (or the resonances) happen quite often between two eigenmodes involving a zero eigenmode. Thus, Assumption 1.7 is natural in such a sense.

\subsubsection{Main result}

We are ready to state our main theorem:

Theorem 1.8. Let $s>d / 2$ and $0<\varepsilon<\varepsilon_{0}$ with $\varepsilon_{0}$ sufficient small. Under Assumption 1.1, Assumption 1.2 and Assumption 1.6, the Cauchy problem (1.1) admits a unique solution $U \in L^{\infty}\left(\left[0, \frac{T_{1}}{\varepsilon^{\alpha}}\right] ; H^{s}\right)$ for some $T_{1}>0$ independent of $\varepsilon$. Moreover, there holds the error estimate

$$
\left\|U-U_{a}\right\|_{L^{\infty}\left(\left[0, \frac{T_{1}}{\varepsilon^{\alpha}}\right] ; H^{s}\right)} \leq C \varepsilon .
$$

If in addition Assumption 1.7 is satisfied, the solution $U \in L^{\infty}\left(\left[0, \frac{T_{2}}{\varepsilon^{\alpha_{1}}}\right] ; H^{s}\right)$ where $T_{2}>0$ is independent of $\varepsilon$ and

$$
\alpha_{1}:=\min \{2 \alpha, 1\} .
$$

Moreover, there holds

$$
\left\|U-U_{a}\right\|_{L^{\infty}\left(\left[0, \frac{T_{2}}{\varepsilon^{\alpha} 1}\right] ; H^{s}\right)} \leq C \varepsilon .
$$

Here $C$ is a constant independent of $\varepsilon$ and the number $\alpha$ is defined in (1.16).

We remark that Theorem 1.8 shows a linear stability phenomenon.

Remark 1.9. Theorem 1.8 gives an existence and stability result beyond the classical existence time. In Assumption 1.2, the initial difference between the exact solution and the approximate solution is of order $O(\varepsilon)$. The estimates (1.17) and (1.18) imply that the error stays of order $O(\varepsilon)$ over long time intervals considered. This means that the approximate solution is linearly stable over the corresponding long time intervals, in the sense that the initial error is not much amplified through the dynamics of the system. 


\subsection{Structure of the paper}

In Section 2, we introduce some context of our study in geometric optics and we also emphasize the novelty of the study in this paper. In Section 3, we recall the concept of semiclassical Fourier multipliers and the action estimates including a commutator estimate. Section 4, Section 5 and Section 6 are devoted to the proof of Theorem 1.8. In Section 7 , we give an application of our study in the non-relativistic limit problem of Klein-Gordon equations.

In the sequel, we use $C$ to denote a positive constant independent of $\varepsilon$. However, the value of $C$ may change from line to line.

\section{Transparency conditions and stabilities}

In this section, we first recall some basic concepts in geometric optics including transparency conditions exhibited in [12] by Joly, Métivier and Rauch, and the normal form method used to obtain the stability of WKB approximate solutions. We then briefly recall the study in [18] and explain why the study of this paper is important for stability analysis in geometric optics, particularly in completing the program of [18] and [12].

\subsection{Weak transparency}

In Section 1.2.2, we gave the definition of WKB solutions and we assumed the existence of WKB solutions in Assumption 1.2. In [11, 12], Joly, Métivier and Rauch exhibited the weak transparency condition that allows one to construct WKB approximate solutions. Before stating such weak transparency condition, we introduce some basic concepts.

We define the characteristic variety of the differential operator in (1.1):

$$
\text { Char }:=\left\{(\tau, \xi): \operatorname{det}\left(-i \tau+A(i \xi)+A_{0}\right)=0\right\} .
$$

Given a couple $(\tau, \xi)$, we denote by $\Pi(\tau, \xi)$ the orthogonal projector onto

$$
\operatorname{ker}\left(-i \tau+A(i \xi)+A_{0}\right)
$$

We fix a basic characteristic space-time vector

$$
\beta:=(\omega, k) \in \mathbb{R} \times \mathbb{R}^{d}
$$

satisfying the dispersion relation

$$
\operatorname{det}\left(-i p \omega+A(i k)+A_{0}\right)=0,
$$

where $k$ is called the spatial wave number and $\omega$ is called the temporal wave number. 
Remark that in this paper, the spatial wave number $k$ is assumed to be zero, since we are not considering solutions that are highly oscillating in spatial variable (see (1.2)). However, to introduce the general concepts concerning transparency conditions, we take general $\beta=(\omega, k)$.

Now we can state the weak transparency condition introduce in [12]:

Weak transparency. For any $p, p_{1} \in \mathbb{Z}$ and any $U, V \in \mathbb{C}^{N}$, one has

$$
\left|\Pi\left(p_{1} \beta\right) B\left(\Pi\left(\left(p_{1}-p\right) \beta\right) U, \Pi(p \beta) V\right)\right|=0 .
$$

We find that this weak transparency condition corresponds to exactly the condition introduced in (1.10).

\subsection{Strong transparency and normal form method}

Given a WKB solution, a nature question is the stability property of this WKB solution. To this issue, one turns to consider the perturbed system. Let $U$ and $U_{a}$ be the exact solution and the WKB solution which solve (1.1) and (1.5) respectively. Then the perturbation

$$
\dot{U}:=\frac{U-U_{a}}{\varepsilon}
$$

solves

$$
\left\{\begin{array}{l}
\partial_{t} \dot{U}+\frac{1}{\varepsilon} A\left(\partial_{x}\right) \dot{U}+\frac{1}{\varepsilon^{2}} A_{0} \dot{U}=2 B\left(U_{a}\right) \dot{U}+\varepsilon B(\dot{U}, \dot{U})+\varepsilon^{K_{a}-1} R^{\varepsilon} \\
\dot{U}(0, x)=\varepsilon^{K-1} \psi^{\varepsilon}(x) .
\end{array}\right.
$$

An advantage of considering the perturbed system (2.3) is that the nonlinear term is small of order $\varepsilon$. The leading term becomes the linear one $2 B\left(U_{a}\right) \dot{U}$. However, even when the parameter $K_{a}$ and $K$ are sufficiently large and the WKB solution $U_{a}$ is uniformly bounded in proper Sobolev spaces and solves (1.5) globally in time, the classical existence time $T_{\varepsilon}^{*}$ to $(2.3)$ is at most of logarithmic order:

$$
\dot{T}_{\varepsilon}^{*} \geq T_{0}|\ln \varepsilon|, \quad \text { for some } T_{0}>0 \text { independent of } \varepsilon \text {. }
$$

This logarithmic order existence time can be achieved by employing the argument in [14] as well as in [4].

To achieve an even larger scale of the maximal existence time such as

$$
\dot{T}_{\varepsilon}^{*} \geq \frac{T}{\varepsilon^{\gamma}}, \quad \text { for some } T>0, \gamma>0 \text { independent of } \varepsilon,
$$

as well as the uniform boundedness of the perturbation over such long time, one needs to make use of more structure of the system (2.3). To this end, also in [12], Joly, Métivier and Rauch introduced the strong transparency condition that allows them to eliminate the linear leading term $2 B\left(U_{a}\right) \dot{U}$ up to a remainder of order $\varepsilon$ 
by using a normal form method. If this can be done, the right-hand side of $(2.3)_{1}$ becomes of order $\varepsilon$ and the well-posedness over time of order $1 / \varepsilon$ follows from the classical theory. We recall the strong transparency condition:

Strong transparency. There exists a constant $C$ such that for any $p \in \mathbb{Z}$, $1 \leq j, j^{\prime} \leq J, \xi \in \mathbb{R}^{d}$ and $U, V \in \mathbb{C}^{N}$, one has

$$
\left|\Pi_{j}(\xi+p k) B\left(\Pi(p \beta) U, \Pi_{j^{\prime}}(\xi) V\right)\right| \leq C\left|\lambda_{j}(\xi+p k)-\lambda_{j^{\prime}}(\xi)-p \omega\right| \cdot|U| \cdot|V| .
$$

In the above inequality $(2.4)$, the terms $\Pi_{j}(\xi+p k) B\left(\Pi(p \beta), \Pi_{j^{\prime}}(\xi)\right)$ on the lefthand side are named interaction coefficients, and the factors $\lambda_{j}(\xi+p k)-\lambda_{j^{\prime}}(\xi)-p \omega$ on the right-hand side are called interaction phases. The frequencies $\xi$ such that $\lambda_{j}(\xi+p k)-\lambda_{j^{\prime}}(\xi)-p \omega=0$ are named resonances and the $\left(j, j^{\prime}, p\right)$-resonance set is defined as

$$
R_{j, j^{\prime}, p}:=\left\{\xi \in \mathbb{R}^{d}, \lambda_{j}(\xi+p k)=p \omega+\lambda_{j^{\prime}}(\xi)\right\}
$$

The equalities $\lambda_{j}(\xi+p k)-\lambda_{j^{\prime}}(\xi)-p \omega=0$ are named resonance equations.

The strong transparency condition offers a control of the quantity

$$
\frac{\Pi_{j}(\xi+p k) B\left(\Pi(p \beta) U_{0, p}, \Pi_{j^{\prime}}(\xi)\right)}{\lambda_{j}(\xi+p k)-\lambda_{j^{\prime}}(\xi)-p \omega}
$$

which appears in the normal form reduction. The interaction phase plays the role of divisor.

The method of a normal form reduction is essentially a change of unknown which can be linear or nonlinear. In general, the nonlinear normal form method needs more constrains on the structure of the equations than the linear one. In our setting, we are trying to eliminate the linear leading term, it is possible to use the linear version of the normal form method. The idea is to consider a change of unknown of the following form

$$
\dot{U}_{1}=\left(\operatorname{Id}+\varepsilon^{2} M\right)^{-1} \dot{U},
$$

with $M$ to be determined. Then the system in $\dot{U}_{1}$ is of the form

$$
\partial_{t} \dot{U}_{1}+\frac{i}{\varepsilon^{2}} \mathcal{A}\left(\varepsilon D_{x}\right) \dot{U}_{1}=\left(2 B\left(U_{a}\right)-i\left[\mathcal{A}\left(\varepsilon D_{x}\right), M\right]\right)+\varepsilon \mathcal{R}_{1},
$$

where $\varepsilon \mathcal{R}_{1}$ contains all the terms formally of order $O(\varepsilon)$ and

$$
D_{x}:=\partial_{x} / i, \quad \mathcal{A}(\xi):=A(\xi)+A_{0} / i .
$$

The goal is to find a proper operator $M$ such that the $O(1)$ term on the right-hand side of (2.6) is eliminated with a small remainder. It is shown in [12], as well as in $[25,16,18,17]$, that such $M$ can be well defined provided the strong transparency 
condition is satisfied, and the linear leading term can be eliminated with an $O(\varepsilon)$ remainder.

Such strong transparency condition is satisfied for some physical models, such as the Maxwell-Bloch system (see [12]) and the one-dimensional Maxwell-LandauLifshitz system (see [16]). Moreover, Texier showed that the Euler-Maxwell equations satisfy a form of transparency [25], Cheverry, Guès and Métivier [2] showed that for systems of conservation laws, linear degeneracy of a field implies transparency. However, many (most) physical models in geometric optics do not fulfill the strong transparency condition, such as the Klein-Gordon system considered in Section 7 later on.

In this paper, we impose a weaker condition in Assumption 1.6 compared to the strong transparency condition. A key novelty of our study is to extend the long time stability analysis in geometric optics under Assumption 1.6 without assuming the strong transparency condition. Another novelty is to introduce a singular localization method, which allows us to do delicate analysis for the interaction coefficients near resonances in order to obtain long time stability.

\subsection{Absence of strong transparency}

In [18], Texier and the first author give a systematic study for the case where the strong transparency condition fails to be satisfied for semilinear hyperbolic systems of the following form:

$$
\partial_{t} U+\frac{1}{\varepsilon} A_{0} U+\sum_{1 \leq j \leq d} A_{j} \partial_{x_{j}} U=\frac{1}{\sqrt{\varepsilon}} B(U, U),
$$

where the constant matrix $A_{0}$ is non-zero and skew-symmetric and the matrices $A_{j}, 1 \leq j \leq d$ are constant and symmetric. Highly oscillating initial data are considered:

$$
U(0, x)=\Re e\left(a(x) e^{i k \cdot x / \varepsilon}\right)+\sqrt{\varepsilon} \varphi^{\varepsilon}(x) .
$$

Here $k$ is the spatial wave number. Let $\omega$ be a temporal wave number satisfying the dispersion relation:

$$
\operatorname{det}\left(-i \omega+A(i k)+A_{0}\right)=0 .
$$

The absence of strong transparency means that there exists $\left(j, j^{\prime}, p\right)$ such that (2.4) is not satisfied. Denote $J_{0}$ the set containing all such indices $\left(j, j^{\prime}, p\right)$ and $R_{j, j^{\prime}, p}$ the $\left(j, j^{\prime}, p\right)$-resonant set defined as in $(2.5)$. If $R_{j, j^{\prime}, p}$ is empty, by the regularity of $\lambda_{j}$ and $\Pi_{j}, j=1, \cdots, J$, the strong transparency condition (2.4) is satisfied for the index $\left(j, j^{\prime}, p\right)$. Then for any $\left(j, j^{\prime}, p\right) \in J_{0}, R_{j, j^{\prime}, p}$ is not empty, and the following quantity is well defined:

$$
\Gamma:=\sup _{\left(j, j^{\prime}, p\right) \in J_{0}}\left|g_{p}\left(0, x_{p}\right)\right|^{2} \sup _{\xi \in R_{j, j^{\prime}, p}} \operatorname{tr}\left(\Pi_{j}(\xi+p k) B\left(\vec{e}_{p}\right) \Pi_{j^{\prime}}(\xi) B\left(\vec{e}_{-p}\right) \Pi_{j}(\xi+p k)\right),
$$


where $g_{p}$ comes from the polarization condition

$$
U_{0, p}(t, x)=g_{p}(t, x) e_{p}, \quad e_{p} \in \operatorname{ker}\left(-i p \omega+A(i k)+A_{0}\right),
$$

and $x_{p}$ is a point where $\left|g_{p}(0, \cdot)\right|$ admits its maximum. Here $U_{0, p}$ are the leading terms of the WKB solution. In [18], it is shown that the stability of the WKB solution is determined by the sign of $\Gamma$ :

If $\Gamma<0$, the perturbation system is symmetrizable and the WKB solution is stable. If $\Gamma>0$, it is shown that the WKB solution is unstable.

However, the degenerate case $\Gamma=0$ is not included in the study of [18]. In [17], the first author considered a subcase of $\Gamma=0$, that is the case $g_{p}(0, x)=0$ for any $\left(j, j^{\prime}, p\right) \in J_{0}$. Under the assumptions $\partial_{t} g_{p}(0, x) \neq 0$ and the positivity of the following quantity

$$
\tilde{\Gamma}:=\sup _{\left(j, j^{\prime}, p\right) \in J_{0}} \sup _{\xi \in R_{j, j^{\prime}, p}} \operatorname{tr}\left(\Pi_{j}(\xi+p k) B\left(\vec{e}_{p}\right) \Pi_{j^{\prime}}(\xi) B\left(\vec{e}_{-p}\right) \Pi_{j}(\xi+p k)\right),
$$

the instability are discovered instantaneously, even though the equations linearized around the leading WKB terms are initially stable.

The study of this paper corresponds to a large subcase of $\Gamma=0$ which goes through the case $\tilde{\Gamma}=0$ under our key Assumption 1.6. Indeed, Assumption 1.6 states that, near resonances, the interaction coefficients $\Pi_{j}(\xi+p k) B\left(\Pi(p \beta), \Pi_{j^{\prime}}(\xi)\right)$ cannot be controlled by the resonant phase $\left|\lambda_{j}(\xi+p k)-\lambda_{j^{\prime}}(\xi)-p \omega\right|$, but rather are controlled by some fraction power of the resonant phase $\mid \lambda_{j}(\xi+p k)-\lambda_{j^{\prime}}(\xi)-$ $\left.p \omega\right|^{\alpha}, 0<\alpha \leq 1$. Even the scaling of this paper is different from that in [18], the idea introduced in this paper may be well employed.

\section{Semiclassical Fourier multipliers}

In this section, we introduce the basic concepts about semiclassical Fourier multipliers, in particular the commutator estimates between a semiclassical Fourier multiplier and a scalar function multiplier. This will be needed throughout the paper. In the sequel of this paper, the function $g$, or $g_{p}$ in the next sections, is also considered as the operator which consists in the multiplication by this function.

We say a smooth scalar, vector or matrix valued function $\sigma(\xi)$ to be a classical symbol of order $m$ provided

$$
\left|\partial_{\xi}^{\alpha} \sigma(\xi)\right| \leq C_{\alpha}\langle\xi\rangle^{m-\alpha}, \quad\langle\xi\rangle:=\left(1+|\xi|^{2}\right)^{\frac{1}{2}}, \quad \text { for any } \alpha \in \mathbb{N}^{d} .
$$

We use $S^{m}$ to denote the set of all classical symbols of order $m$. The classical Fourier multiplier associated with a symbol $\sigma(\xi)$ is denoted by $\sigma\left(D_{x}\right)$, and is defined as

$$
\sigma\left(D_{x}\right) u:=\mathfrak{F}^{-1}[\sigma(\xi) \hat{u}(\xi)]=\mathfrak{F}^{-1}[\sigma] * u,
$$


where $\hat{u}(\xi)=\mathfrak{F}[u](\xi)$ is the Fourier transform of $u$ and $\mathfrak{F}^{-1}$ denotes the inverse of Fourier transform.

The semiclassical Fourier multiplier associated with a symbol $\sigma(\xi)$ is denoted by $\sigma\left(\varepsilon D_{x}\right)$, and is defined as

$$
\sigma\left(\varepsilon D_{x}\right) u:=\mathfrak{F}^{-1}[\sigma(\varepsilon \xi) \hat{u}(\xi)]=\mathfrak{F}^{-1}[\sigma(\varepsilon \cdot)] * u=\varepsilon^{-d} \mathfrak{F}^{-1}[\sigma]\left(\frac{\dot{\varepsilon}}{\varepsilon}\right) * u .
$$

The definitions in (3.1) and (3.2) can be generated to less regular symbols $\sigma$ as long as the definitions make sense.

We now give two properties that we will use in this paper for classical and semiclassical Fourier multipliers. The first one is rather direct:

Lemma 3.1. Let $\sigma \in L^{\infty}$, then for any $s \in \mathbb{R}$ and $\varepsilon>0$ :

$$
\left\|\sigma\left(D_{x}\right) u\right\|_{H^{s}} \leq\|\sigma(\cdot)\|_{L^{\infty}}\|u\|_{H^{s}}, \quad\left\|\sigma\left(\varepsilon D_{x}\right) u\right\|_{H^{s}} \leq\|\sigma(\cdot)\|_{L^{\infty}}\|u\|_{H^{s}} .
$$

The second one is about the commutator estimates.

Lemma 3.2. Let $\sigma \in C^{1}$ such that $\left\|\nabla_{\xi} \sigma\right\|_{L^{\infty}}<\infty$ and $g(x) \in H^{d / 2+1+\eta_{0}}$ a scalar function for some $\eta_{0}>0$. Then there holds for any $s \geq 0$ :

$$
\left\|\left[\sigma\left(\varepsilon D_{x}\right), g(x)\right] u\right\|_{H^{s}} \leq \varepsilon C_{\eta_{0}} 2^{s}\left\|\nabla_{\xi} \sigma\right\|_{L^{\infty}}\left(\|g\|_{H^{\frac{d}{2}+1+\eta_{0}}}\|u\|_{H^{s}}+\|g\|_{H^{s+1}}\|u\|_{H^{\frac{d}{2}+\eta_{0}}}\right) .
$$

The point of Lemma 3.2 is that the commutator of a semiclassical Fourier multiplier and a regular scalar function is of order $\varepsilon$.

Proof of Lemma 3.2. Let

$$
I(\xi):=\mathfrak{F}\left[\left[\sigma\left(\varepsilon D_{x}\right), g(x)\right] u\right](\xi) .
$$

Then

$$
\left\|\left[\sigma\left(\varepsilon D_{x}\right), g(x)\right] u\right\|_{H^{s}}=\left\|\langle\xi\rangle^{s} I(\xi)\right\|_{L^{2}} .
$$

By the definition of semiclassical Fourier multiplier, we have

$$
\begin{aligned}
I(\xi) & =\mathfrak{F}\left[\sigma\left(\varepsilon D_{x}\right)(g u)\right]-\mathfrak{F}\left[g \sigma\left(\varepsilon D_{x}\right)(u)\right]=\sigma(\varepsilon \xi) \mathfrak{F}[(g u)]-\mathfrak{F}\left[g \sigma\left(\varepsilon D_{x}\right)(u)\right] \\
& =\sigma(\varepsilon \xi)(\hat{g} * \hat{u})(\xi)-(\hat{g} *(\sigma(\varepsilon \cdot) \hat{u})(\xi) \\
& =\sigma(\varepsilon \xi) \int_{\mathbb{R}^{d}} \hat{g}(\eta) \hat{u}(\xi-\eta) d \eta-\int_{\mathbb{R}^{d}} \hat{g}(\eta) \sigma(\varepsilon \xi-\varepsilon \eta) \hat{u}(\xi-\eta) d \eta \\
& =\int_{\mathbb{R}^{d}} \hat{g}(\eta)(\sigma(\varepsilon \xi)-\sigma(\varepsilon \xi-\varepsilon \eta)) \hat{u}(\xi-\eta) d \eta \\
& =\int_{\mathbb{R}^{d}} \hat{g}(\eta) \int_{0}^{1} \varepsilon \eta \cdot\left(\nabla_{\xi} \sigma\right)(\varepsilon \xi-\varepsilon(1-t) \eta) d t \hat{u}(\xi-\eta) d \eta .
\end{aligned}
$$


Then

$$
\begin{aligned}
&\left|\langle\xi\rangle^{s} I(\xi)\right| \leq \varepsilon\left\|\nabla_{\xi} \sigma\right\|_{L^{\infty}} \int_{\mathbb{R}^{d}}\langle\xi\rangle^{s}|\eta||\hat{g}(\eta)||\hat{u}(\xi-\eta)| d \eta \\
& \leq \varepsilon\left\|\nabla_{\xi} \sigma\right\|_{L^{\infty}}\left(\int_{|\eta|>\frac{|\xi|}{2}}\langle\xi\rangle^{s}|\eta||\hat{g}(\eta)||\hat{u}(\xi-\eta)| d \eta\right. \\
&\left.\quad+\int_{|\eta| \leq \frac{|\xi|}{2}}\langle\xi\rangle^{s}|\eta||\hat{g}(\eta)||\hat{u}(\xi-\eta)| d \eta\right) \\
& \leq \varepsilon 2^{s}\left\|\nabla_{\xi} \sigma\right\|_{L^{\infty}}\left(\int_{|\eta|>\frac{|\xi|}{2}}\langle\eta\rangle^{s}|\eta||\hat{g}(\eta)||\hat{u}(\xi-\eta)| d \eta\right. \\
&\left.\quad+\int_{|\eta| \leq \frac{|\xi|}{2}}\langle\xi-\eta\rangle^{s}|\eta||\hat{g}(\eta)||\hat{u}(\xi-\eta)| d \eta\right) \\
& \leq \varepsilon 2^{s}\left\|\nabla_{\xi} \sigma\right\|_{L^{\infty}}\left(\left|\langle\xi\rangle^{s+1} \hat{g}(\xi)\right| *|\hat{u}(\xi)|+|\xi \hat{g}(\xi)| *\left|\langle\xi\rangle^{s} \hat{u}(\xi)\right|\right) .
\end{aligned}
$$

Young's inequality yields

$$
\left|\langle\xi\rangle^{s} I(\xi)\right|_{L^{2}} \leq \varepsilon 2^{s}\left\|\nabla_{\xi} \sigma\right\|_{L^{\infty}}\left(\left\|\langle\xi\rangle^{s+1} \hat{g}(\xi)\right\|_{L^{2}}\|\hat{u}(\xi)\|_{L^{1}}+\|\xi \hat{g}(\xi)\|_{L^{1}}\left\|\langle\xi\rangle^{s} \hat{u}(\xi)\right\|_{L^{2}}\right) .
$$

Hölder's inequality implies

$$
\|\xi \hat{g}(\xi)\|_{L^{1}} \leq C_{\eta_{0}}\left\|\langle\xi\rangle^{d / 2+1+\eta_{0}} \hat{g}(\xi)\right\|_{L^{2}}, \quad\|\hat{u}(\xi)\|_{L^{1}} \leq C_{\eta_{0}}\left\|\langle\xi\rangle^{d / 2+\eta_{0}} \hat{u}(\xi)\right\|_{L^{2}} .
$$

Finally, we obtain

$$
\left|\langle\xi\rangle^{s} I(\xi)\right|_{L^{2}} \leq \varepsilon C_{\eta_{0}} 2^{s}\left\|\nabla_{\xi} \sigma\right\|_{L^{\infty}}\left(\|g\|_{H^{\frac{d}{2}+1+\eta_{0}}}\|u\|_{H^{s}}+\|g\|_{H^{s+1}}\|u\|_{H^{\frac{d}{2}+\eta_{0}}}\right) .
$$

This completes the proof of Lemma 3.2 .

\section{Perturbed system and diagonalization}

Now we start proving Theorem 1.8. From now on, we suppose Assumption 1.1, Assumption 1.2 and Assumption 1.6 are satisfied.

\subsection{Perturbed system near approximate solution}

Associated with the approximate solution $U_{a}$ given in Assumption 1.2, we define the perturbation

$$
\dot{U}:=\frac{U-U_{a}}{\varepsilon},
$$

where $U \in C\left(\left[0, T_{\varepsilon}^{*}\right) ; H^{s}\right)$ is the local-in-time solution to original Cauchy problem (1.1). Then at least over time interval $\left[0, T_{\varepsilon}^{*}\right)$, the perturbation $\dot{U}$ solves

$$
\left\{\begin{array}{l}
\partial_{t} \dot{U}+\frac{1}{\varepsilon} A\left(\partial_{x}\right) \dot{U}+\frac{1}{\varepsilon^{2}} A_{0} \dot{U}=2 B\left(U_{a}\right) \dot{U}+\varepsilon B(\dot{U}, \dot{U})+\varepsilon R^{\varepsilon}, \\
\dot{U}(0)=\psi^{\varepsilon}
\end{array}\right.
$$


where the linear operator $B\left(U_{a}\right)$ is defined as

$$
B\left(U_{a}\right) W:=B\left(U_{a}, W\right), \quad \text { for any } W \in \mathbb{C}^{N} .
$$

The remainder $\left(R^{\varepsilon}, \psi^{\varepsilon}\right)$ satisfies the uniform estimate given in (1.6).

To prove Theorem 1.8, it is sufficient to show the existence and uniform estimates for the solution of (4.2) over corresponding long time intervals.

The perturbed system (4.2) has small nonlinearity of order $O(\varepsilon)$. By careful, rather classical analysis ( $L^{2}$ estimate and Grownwall's inequality), it can be shown that the maximal existence time, denoted by $\dot{T}_{\varepsilon}^{*}$, to Cauchy problem (4.2) satisfies

$$
\lim _{\varepsilon \rightarrow 0} \dot{T}_{\varepsilon}^{*}=\infty .
$$

By employing the argument in [4], one can even show the existence up to time of the logarithmic order:

$$
\dot{T}_{\varepsilon}^{*} \geq T_{0}|\ln \varepsilon|, \quad \text { for some } T_{0}>0 \text { independent of } \varepsilon .
$$

To show the existence up to even longer time of order $O\left(1 / \varepsilon^{\gamma}\right)$, we need to discover more structure of the system (4.2). To this end, we will diagonalize the differential operator on the left-hand side of (4.2) by diagonalizing the corresponding symbol, then consider the system mode by mode.

\subsection{Diagonalization}

According to the smooth spectral decomposition assumed in Assumption 1.1, we can write

$$
A\left(\varepsilon D_{x}\right)+A_{0} / i=\sum_{j=1}^{J} \lambda_{j}\left(\varepsilon D_{x}\right) \Pi_{j}\left(\varepsilon D_{x}\right), \quad D_{x}:=\partial_{x} / i .
$$

We want to go deep to the structure of the system in (4.2). Hence, we consider the system mode by mode, through the following change of unknown:

$$
\dot{U}_{1}=\left(\begin{array}{c}
\dot{U}_{1}^{1} \\
\vdots \\
\dot{U}_{1}^{J}
\end{array}\right):=\left(\begin{array}{c}
\Pi_{1}\left(\varepsilon D_{x}\right) \dot{U} \\
\vdots \\
\Pi_{J}\left(\varepsilon D_{x}\right) \dot{U}
\end{array}\right) \in \mathbb{R}^{J N}
$$

We remark that, by Lemma $3.1, \Pi_{j}\left(\varepsilon D_{x}\right), 1 \leq j \leq J$ are linear operators bounded from $H^{s}$ to $H^{s}$ for any $s \in \mathbb{R}$. Hence

$$
\left\|\dot{U}_{1}(t, \cdot)\right\|_{H^{s}} \leq C\|\dot{U}(t, \cdot)\|_{H^{s}}, \quad \text { for any } s \in \mathbb{R} \text { and any } t \geq 0 .
$$

Conversely, we can reconstruct $\dot{U}$ via $\dot{U}_{1}$ :

$$
\dot{U}:=\sum_{j=1}^{J} U_{1}^{j}
$$


due to the fact

$$
\sum_{j=1}^{J} \Pi_{j}=\mathrm{Id}
$$

We observe that

$$
B\left(U_{a}\right)=B\left(U_{0}\right)+\varepsilon B\left(U_{r}\right), \quad U_{r}:=U_{1}+\varepsilon U_{2}+\varepsilon^{2} U_{3} .
$$

Then by (4.2), the equation in $\dot{U}_{1}$ is of the form

$$
\partial_{t} \dot{U}_{1}+\frac{i}{\varepsilon^{2}} A_{1}\left(\varepsilon D_{x}\right) \dot{U}_{1}=B_{1} \dot{U}_{1}+\varepsilon B_{r} \dot{U}_{1}+\varepsilon F_{1}\left(\dot{U}_{1}, \dot{U}_{1}\right)+\varepsilon R_{1} .
$$

The propagator $A_{1}$ on the left-hand side is a diagonal matrix valued semiclassical Fourier multiplier

$$
A_{1}\left(\varepsilon D_{x}\right):=\operatorname{diag}\left\{\lambda_{1}\left(\varepsilon D_{x}\right), \cdots, \lambda_{J}\left(\varepsilon D_{x}\right)\right\} .
$$

The leading linear operator $B_{1}$ on the right-hand side is

$$
B_{1}:=2\left(\Pi_{j}\left(\varepsilon D_{x}\right) B\left(U_{0}\right) \Pi_{j^{\prime}}\left(\varepsilon D_{x}\right)\right)_{1 \leq j, j^{\prime} \leq J},
$$

which is of matrix form and is associated with the leading term $U_{0}$. By the form of $U_{0}$ in Assumption 1.2, we have

$$
\Pi_{j}\left(\varepsilon D_{x}\right) B\left(U_{0}\right) \Pi_{j^{\prime}}\left(\varepsilon D_{x}\right):=\sum_{p= \pm 1} e^{-i p \omega t / \varepsilon^{2}} \Pi_{j}\left(\varepsilon D_{x}\right) B\left(U_{0, p}\right) \Pi_{j^{\prime}}\left(\varepsilon D_{x}\right) .
$$

The terms $\Pi_{j}\left(\varepsilon D_{x}\right) B\left(U_{0, p}\right) \Pi_{j^{\prime}}\left(\varepsilon D_{x}\right)$ are also named interaction coefficients. To specify, $\Pi_{j}\left(\varepsilon D_{x}\right) B\left(U_{0, p}\right) \Pi_{j^{\prime}}\left(\varepsilon D_{x}\right)$ is called $\left(j, j^{\prime}, p\right)$ interaction coefficient.

The remainder linear operator $B_{r}$ is

$$
B_{r}:=2\left(\Pi_{j}\left(\varepsilon D_{x}\right) B\left(U_{r}\right) \Pi_{j^{\prime}}\left(\varepsilon D_{x}\right)\right)_{1 \leq j, j^{\prime} \leq J},
$$

which is associated with the remainder term $U_{r}$ defined in (4.4).

The nonlinear term $F_{1}$ is

$$
F_{1}\left(\dot{U}_{1}, \dot{U}_{1}\right):=\left(\begin{array}{c}
\Pi_{1}\left(\varepsilon D_{x}\right) B(\dot{U}, \dot{U}) \\
\vdots \\
\Pi_{J}\left(\varepsilon D_{x}\right) B(\dot{U}, \dot{U})
\end{array}\right), \quad \dot{U}=\sum_{j=1}^{J} \dot{U}_{1}^{j} .
$$

Finally the remainder $R_{1}$ is

$$
R_{1}:=\left(\begin{array}{c}
\Pi_{1}\left(\varepsilon D_{x}\right) R^{\varepsilon} \\
\vdots \\
\Pi_{J}\left(\varepsilon D_{x}\right) R^{\varepsilon}
\end{array}\right)
$$


To avoid notational complexity, we rewrite (4.5) in the following more compact form

$$
\partial_{t} \dot{U}_{1}+\frac{i}{\varepsilon^{2}} A_{1}\left(\varepsilon D_{x}\right) \dot{U}_{1}=B_{1} \dot{U}_{1}+\varepsilon \mathcal{R}_{1},
$$

where $\mathcal{R}_{1}$ is the sum of all the $O(\varepsilon)$ terms. By the uniform estimates for the approximate solution assumed in Assumption 1.2, and by Lemma 3.1 and Lemma 3.2 about the actions of Fourier multipliers, we have the estimate

$$
\left\|\mathcal{R}_{1}(t, \cdot)\right\|_{H^{\mu}} \leq C\left(1+\|\dot{U}(t, \cdot)\|_{L^{\infty}}\right)\|\dot{U}(t, \cdot)\|_{H^{\mu}}, \quad \text { for all } 0 \leq \mu \leq s .
$$

The initial datum of $\dot{U}_{1}$ is

$$
\dot{U}_{1}(0)=\left(\begin{array}{c}
\Pi_{1}\left(\varepsilon D_{x}\right) \psi^{\varepsilon} \\
\vdots \\
\Pi_{J}\left(\varepsilon D_{x}\right) \psi^{\varepsilon}
\end{array}\right),
$$

which is uniformly bounded in $H^{s}$.

\section{$5 \quad$ Long time stability: Part I}

This section is devoted to proving the first part of Theorem 1.8, that is the stability over time of order $O\left(1 / \varepsilon^{\alpha}\right)$ under Assumption 1.1, Assumption 1.2 and Assumption 1.6 .

\subsection{A singular localization}

To show long time of order $O\left(1 / \varepsilon^{\alpha}\right)$ well-posedness for (4.8) with $O(1)$ initial datum (4.9), the idea here is to eliminate the $O(1)$ term $B_{1}$ on the right-hand side of (4.8) up to a small remainder of order $O\left(\varepsilon^{\alpha}\right)$ in this section. Then we employ the classical theory to obtain the long time existence. However, the strong transparency condition is not satisfied in our setting, so we cannot simply use the normal form reduction method to achieve this. The main novelty of our study is to carry out a singular localization on the interaction coefficients; together with the normal form reduction, we show that we can eliminate the $O(1)$ interaction coefficients up to small remainders.

We recall the definition of resonance sets for any $1 \leq j \leq J, 1 \leq j^{\prime} \leq J, p \in$ $\{-1,1\}$ in Assumption 1.6:

$$
R_{j, j^{\prime}, p}:=\left\{\xi \in \mathbb{R}^{d}: \lambda_{j}(\xi)-\lambda_{j^{\prime}}(\xi)-p \omega=0\right\} .
$$

Compared to the definition in (2.5), we remark that here we have the zero spatial wave number: $k=0$. 
If for some $\left(j^{\prime}, j, p\right)$ the corresponding resonance set $R_{j, j^{\prime}, p}$ is empty, by Assumption 1.6 and the smoothness and boundedness of $\Pi_{j}(\cdot)$, the following strong transparency condition is automatically satisfied:

$$
\left|\Pi_{j}(\xi) B\left(e_{p}\right) \Pi_{j^{\prime}}(\xi)\right| \leq C\left|\lambda_{j}(\xi)-\lambda_{j^{\prime}}(\xi)-p \omega\right| .
$$

This indicates that, if $\left(j, j^{\prime}, p\right) \in J_{r}$ defined as

$$
J_{r}:=\left\{\left(j, j^{\prime}, p\right): R_{j, j^{\prime}, p}=\emptyset\right\},
$$

the exponent $\alpha_{j, j^{\prime}, p}$ in (1.13) can be taken to be 1 . We thus introduce the index set

$$
J_{1}:=\left\{\left(j, j^{\prime}, p\right):(1.13) \text { holds for } \alpha_{j, j^{\prime}, p}=1\right\} \supset J_{r} .
$$

Now we introduce smooth cut-off functions $\chi_{j, j^{\prime}, p}$ that are supported near resonance sets:

$$
\begin{aligned}
& \text { If }\left(j, j^{\prime}, p\right) \in J_{1}, \quad \chi_{j, j^{\prime}, p} \equiv 0 . \\
& \text { If }\left(j, j^{\prime}, p\right) \notin J_{1}, \quad \chi_{j, j^{\prime}, p} \in C_{c}^{\infty}\left(R_{j, j^{\prime}, p}^{2 h_{\varepsilon}}\right), \quad \chi_{j, j^{\prime}, p} \equiv 1 \text { on } R_{j, j^{\prime}, p}^{h_{\varepsilon}}, \quad 0 \leq \chi_{j, j^{\prime}, p} \leq 1,
\end{aligned}
$$

where $0<h_{\varepsilon}<1$ is a small positive number depending on $\varepsilon$ and is to be determined later on, and

$$
R_{j, j^{\prime}, p}^{h}:=\left\{\xi \in \mathbb{R}^{d}:\left|\lambda_{j}(\xi)-\lambda_{j^{\prime}}(\xi)-p \omega\right|<h\right\}, \quad \text { for } h>0 \text { small. }
$$

By the compactness assumption on resonance sets in Assumption 1.6, for sufficient small $h$, the sets $R_{j, j^{\prime}, p}^{h}$ defined in (5.3) are uniformly bounded. The index $h_{\varepsilon}$ will be chosen relatively small such that $R_{j, j^{\prime}, p}^{2 h_{\varepsilon}}$ are all bounded.

We consider the decomposition of the leading linear operator $B_{1}$ defined in (4.6) and (4.7):

$$
B_{1}:=B_{\text {in }}+B_{\text {out }}
$$

with

$$
\begin{aligned}
& B_{\text {in }}:=2 \sum_{p= \pm 1} e^{-i p \omega t / \varepsilon^{2}}\left(\chi_{j, j^{\prime}, p}\left(\varepsilon D_{x}\right) \Pi_{j}\left(\varepsilon D_{x}\right) B\left(U_{0, p}\right) \Pi_{j^{\prime}}\left(\varepsilon D_{x}\right)\right)_{1 \leq j, j^{\prime} \leq J}, \\
& B_{\text {out }}:=2 \sum_{p= \pm 1} e^{-i p \omega t / \varepsilon^{2}}\left(\left(1-\chi_{j, j^{\prime}, p}\right)\left(\varepsilon D_{x}\right) \Pi_{j}\left(\varepsilon D_{x}\right) B\left(U_{0, p}\right) \Pi_{j^{\prime}}\left(\varepsilon D_{x}\right)\right)_{1 \leq j, j^{\prime} \leq J} .
\end{aligned}
$$

The part $B_{\text {in }}$ is localized near the resonances while the other part $B_{\text {out }}$ is localized away from the resonances. However, this localization depends on $\chi_{j, j^{\prime}, p}$ which may be singular in $\varepsilon$ duce to the definition in (5.2). Indeed, by (5.2), the support of $\chi_{j, j^{\prime}, p}$ shrinks to the resonance set $R_{j, j^{\prime}, p}$ if $h_{\varepsilon} \rightarrow 0$ as $\varepsilon \rightarrow 0$. By our choice of $h_{\varepsilon}$ later on 
(see (5.24) and (6.15)), we do have $h_{\varepsilon} \rightarrow 0$ as $\varepsilon \rightarrow 0$. This causes the derivatives of $\chi_{j, j^{\prime}, p}$ could be unbounded as $\varepsilon \rightarrow 0$. This is why we call this localization to be singular.

First of all, we show that under Assumption 1.6, the part $B_{\text {in }}$ near the resonance is small of order $O\left(h_{\varepsilon}^{\alpha}\right)$ :

Proposition 5.1. There exits $C>0$ such that for any $d / 2<\mu \leq s$ and any $V \in H^{\mu}$, there holds

$$
\left\|B_{i n} V\right\|_{H^{\mu}} \leq C\left(h_{\varepsilon}^{\alpha}+\varepsilon\right)\|V\|_{H^{\mu}} .
$$

Proof of Proposition 5.1. By the definition of $\chi_{j, j^{\prime}, p}$ in (5.2), it is sufficient to prove for any $\left(j, j^{\prime}, p\right) \notin J_{1}$ and any $u \in H^{\mu}$, there holds

$$
\left\|\chi_{j, j^{\prime}, p}\left(\varepsilon D_{x}\right) \Pi_{j}\left(\varepsilon D_{x}\right) B\left(U_{0, p}\right) \Pi_{j^{\prime}}\left(\varepsilon D_{x}\right) u\right\|_{H^{\mu}} \leq C\left(h_{\varepsilon}^{\alpha}+\varepsilon\right)\|u\|_{H^{\mu}} .
$$

By using (1.7) and (1.8) in Assumption 1.2 and the actions of semiclassical Fourier multiplier in Lemma 3.1, we compute

$$
\begin{aligned}
& \left\|\chi_{j, j^{\prime}, p}\left(\varepsilon D_{x}\right) \Pi_{j}\left(\varepsilon D_{x}\right) B\left(U_{0, p}\right) \Pi_{j^{\prime}}\left(\varepsilon D_{x}\right) u\right\|_{H^{\mu}} \\
& =\left\|\chi_{j, j^{\prime}, p}\left(\varepsilon D_{x}\right) \Pi_{j}\left(\varepsilon D_{x}\right) B\left(g_{p} e_{p}\right) \Pi_{j^{\prime}}\left(\varepsilon D_{x}\right) u\right\|_{H^{\mu}} \\
& \leq \\
& \quad\left\|\chi_{j, j^{\prime}, p}\left(\varepsilon D_{x}\right) \Pi_{j}\left(\varepsilon D_{x}\right) B\left(e_{p}\right) \Pi_{j^{\prime}}\left(\varepsilon D_{x}\right)\left(g_{p} u\right)\right\|_{H^{\mu}} \\
& \quad+\left\|\chi_{j, j^{\prime}, p}\left(\varepsilon D_{x}\right) \Pi_{j}\left(\varepsilon D_{x}\right) B\left(e_{p}\right)\left[g_{p}, \Pi_{j^{\prime}}\left(\varepsilon D_{x}\right)\right] u\right\|_{H^{\mu}} \\
& \left.\leq \| \chi_{j, j^{\prime}, p}(\xi) \Pi_{j}(\xi) B\left(e_{p}\right) \Pi_{j^{\prime}}(\xi)\right)\left\|_{L_{\xi}^{\infty}}\right\| g_{p} u \|_{H^{\mu}} \\
& \quad+\left\|\chi_{j, j^{\prime}, p}(\xi) \Pi_{j}(\xi) B\left(e_{p}\right)\right\|_{L_{\xi}^{\infty}}\left\|\left[g_{p}, \Pi_{j^{\prime}}\left(\varepsilon D_{x}\right)\right] u\right\|_{H^{\mu}} .
\end{aligned}
$$

By the definition of $\chi_{j, j^{\prime}, p}$ in (5.2) and the condition (1.13) in Assumption 1.2, we have

$$
\left.\| \chi_{j, j^{\prime}, p}(\xi) \Pi_{j}(\xi) B\left(e_{p}\right) \Pi_{j^{\prime}}(\xi)\right)\left\|_{L_{\xi}^{\infty}} \leq C h_{\varepsilon}^{\alpha}, \quad\right\| \chi_{j, j^{\prime}, p}(\xi) \Pi_{j}(\xi) B\left(e_{p}\right) \|_{L_{\xi}^{\infty}} \leq C .
$$
holds

By the regularity assumption $g_{p} \in L^{\infty}\left(0, \infty ; H^{s+1}\right)$ in Assumption 1.2, there

$$
\left\|g_{p} u\right\|_{H^{\mu}} \leq\left\|g_{p}\right\|_{H^{\mu}}\|u\|_{H^{\mu}} \leq C\|u\|_{H^{\mu}} .
$$

By Lemma 3.2 concerning the commutator estimate, we have

$$
\left\|\left[g_{p}, \Pi_{j^{\prime}}\left(\varepsilon D_{x}\right)\right] u\right\|_{H^{\mu}} \leq C \varepsilon\left\|g_{p}\right\|_{H^{\mu+1}}\|u\|_{H^{\mu}} \leq C \varepsilon\|u\|_{H^{\mu}} .
$$

Combining the estimates in (5.7)-(5.8) implies (5.6). The proof is completed.

Now it is left to deal with the part localized away from resonance sets. 


\subsection{Normal form reduction}

Since $B_{\text {out }}$ is localized away from resonance, we can employ the normal reduction method to eliminate it up to some remainder. The issue is that due the singularity of the localization functions $\chi_{j, j^{\prime}, p}$ in $\varepsilon$, the remainder may not be small.

We will see later on, we can choose $h_{\varepsilon}$ properly to achieve a small remainder. We need to also choose $h_{\varepsilon}$ such that the remainder $B_{\text {out }}$ after the normal form reduction is of the same order as $B_{i n}$ obtained in Proposition 5.1 in order to obtain the minimum remainder.

We introduce the following formal change of unknown

$$
\dot{U}_{2}=\left(\operatorname{Id}+\varepsilon^{2} M\right)^{-1} \dot{U}_{1},
$$

for some operator $M$ of the form

$$
M=\sum_{p= \pm 1} e^{-i p \omega t / \varepsilon^{2}}\left(M_{j j^{\prime}}^{(p)}\right)_{1 \leq j, j^{\prime} \leq J}
$$

with the operator elements $M_{j j^{\prime}}^{(p)}$ to be determined.

Then, by (4.8), the system in $\dot{U}_{2}$ has the form

$$
\begin{aligned}
\partial_{t} \dot{U}_{2} & +\frac{i}{\varepsilon^{2}} A_{1}\left(\varepsilon D_{x}\right) \dot{U}_{2}=\left(\operatorname{Id}+\varepsilon^{2} M\right)^{-1}\left(B_{\text {out }}-i\left[A_{1}\left(\varepsilon D_{x}\right), M\right]-\varepsilon^{2} \partial_{t} M\right) \dot{U}_{2} \\
& +\left(\operatorname{Id}+\varepsilon^{2} M\right)^{-1}\left(\varepsilon^{2} B_{\text {out }} M \dot{U}_{2}+B_{\text {in }}\left(\mathrm{Id}+\varepsilon^{2} M\right) \dot{U}_{2}+\varepsilon \mathcal{R}_{1}\right) .
\end{aligned}
$$

The idea is to find some operator $M$ properly such that the $O(1)$ term on the right-hand side of (5.11) is eliminated with a small remainder. This is done in the following proposition.

Proposition 5.2. There exist symbols $\widetilde{M}_{j j^{\prime}}^{(p)}(\xi) \in S^{0}, 1 \leq j, j^{\prime} \leq J, p \in\{-1,1\}$, such that $M$ defined in (5.10) with $M_{j j^{\prime}}^{(p)}:=\widetilde{M}_{j j^{\prime}}^{(p)}\left(\varepsilon D_{x}\right) \circ g_{p}$, which denotes the composition of Fourier multiplier $\widetilde{M}_{j j^{\prime}}^{(p)}\left(\varepsilon D_{x}\right)$ and function multiplier $g_{p}$, satisfies

$$
B_{\text {out }}-i\left[A_{1}\left(\varepsilon D_{x}\right), M\right]-\varepsilon^{2} \partial_{t} M=\left(\varepsilon h_{\varepsilon}^{\alpha-1}+\varepsilon^{2} h_{\varepsilon}^{\alpha-1}+\varepsilon\right) M_{r},
$$

where $M_{r}$ is a linear operator satisfying the estimate:

$$
\left\|M_{r} V\right\|_{H^{\mu}} \leq C\|V\|_{H^{\mu}}, \quad \text { for any } d / 2<\mu \leq s \text { and any } V \in H^{\mu} .
$$

Proof of Proposition 5.2. Given $M$ of the form (5.10), we compute

$$
\begin{aligned}
& {\left[A_{1}\left(\varepsilon D_{x}\right), M\right]=A_{1}\left(\varepsilon D_{x}\right) M-M A_{1}\left(\varepsilon D_{x}\right)} \\
& =\sum_{p= \pm 1} e^{-i p \omega t / \varepsilon^{2}}\left(\lambda_{j} M_{j j^{\prime}}^{(p)}-M_{j j^{\prime}}^{(p)} \lambda_{j^{\prime}}\right)_{1 \leq j, j^{\prime} \leq J} \\
& =\sum_{p= \pm 1} e^{-i p \omega t / \varepsilon^{2}}\left(\left(\lambda_{j}-\lambda_{j^{\prime}}\right) M_{j j^{\prime}}^{(p)}\right)_{1 \leq j, j^{\prime} \leq J}+M_{r}^{(1)}
\end{aligned}
$$


where we used the simplified notation $\lambda_{j}:=\lambda_{j}\left(\varepsilon D_{x}\right), j \in\{1, \cdots, J\}$ and

$$
M_{r}^{(1)}:=\sum_{p= \pm 1} e^{-i p \omega t / \varepsilon^{2}}\left(\left[\lambda_{j^{\prime}}, M_{j j^{\prime}}^{(p)}\right]\right)_{1 \leq j, j^{\prime} \leq J} .
$$

We then compute

$$
\varepsilon^{2} \partial_{t} M=\sum_{p= \pm 1} e^{-i p \omega t / \varepsilon^{2}}(-i p \omega)\left(M_{j j^{\prime}}^{(p)}\right)_{1 \leq j, j^{\prime} \leq J}+M_{r}^{(2)}
$$

where

$$
M_{r}^{(2)}:=\varepsilon^{2} \sum_{p= \pm 1} e^{-i p \omega t / \varepsilon^{2}}\left(\partial_{t} M_{j j^{\prime}}^{(p)}\right)_{1 \leq j, j^{\prime} \leq J}
$$

Then

$$
\begin{aligned}
& i\left[A_{1}\left(\varepsilon D_{x}\right), M\right]+\varepsilon^{2} \partial_{t} M \\
& =\sum_{p= \pm 1} e^{-i p \omega t / \varepsilon^{2}}\left(i\left(\lambda_{j}-\lambda_{j^{\prime}}-p \omega\right) M_{j j^{\prime}}^{(p)}\right)_{1 \leq j, j^{\prime} \leq J}+M_{r}^{(1)}+M_{r}^{(2)} .
\end{aligned}
$$

By (5.5) and Assumption 1.2, we have

$$
\begin{aligned}
& B_{\text {out }}=2 \sum_{p= \pm 1} e^{-i p \omega t / \varepsilon^{2}}\left(\left(1-\chi_{j, j^{\prime}, p}\right)\left(\varepsilon D_{x}\right) \Pi_{j}\left(\varepsilon D_{x}\right) B\left(U_{0, p}\right) \Pi_{j^{\prime}}\left(\varepsilon D_{x}\right)\right)_{1 \leq j, j^{\prime} \leq J} \\
& =2 \sum_{p= \pm 1} e^{-i p \omega t / \varepsilon^{2}}\left(\left(1-\chi_{j, j^{\prime}, p}\right)\left(\varepsilon D_{x}\right) \Pi_{j}\left(\varepsilon D_{x}\right) B\left(e_{p}\right) \Pi_{j^{\prime}}\left(\varepsilon D_{x}\right) \circ g_{p}\right)_{1 \leq j, j^{\prime} \leq J}+M_{r}^{(3)}
\end{aligned}
$$

with

$$
M_{r}^{(3)}:=2 \sum_{p= \pm 1} e^{-i p \omega t / \varepsilon^{2}}\left(1-\chi_{j, j^{\prime}, p}\right)\left(\varepsilon D_{x}\right) \Pi_{j}\left(\varepsilon D_{x}\right) B\left(e_{p}\right)\left[g_{p}, \Pi_{j^{\prime}}\left(\varepsilon D_{x}\right)\right] .
$$

Now we are ready to give the definitions of $\widetilde{M}_{j j^{\prime}}^{(p)}(\xi)$ :

$$
\widetilde{M}_{j j^{\prime}}^{(p)}(\xi):=-2 i\left(\lambda_{j}(\xi)-\lambda_{j^{\prime}}(\xi)-p \omega\right)^{-1}\left(1-\chi_{j, j^{\prime}, p}\right)(\xi) \Pi_{j}(\xi) B\left(e_{p}\right) \Pi_{j^{\prime}}(\xi) .
$$

We observe that such $\widetilde{M}_{j j^{\prime}}^{(p)}(\xi)$ are well defined due to the localization away from resonances (see (5.2) and (5.3)). Moreover, by the condition (1.13) in Assumption 1.6 and the definition of the cut-off functions in (5.2), we have

$$
\left\|\widetilde{M}_{j j^{\prime}}^{(p)}(\xi)\right\|_{L_{\xi}^{\infty}} \leq C h_{\varepsilon}^{\alpha_{j, j^{\prime}, p^{\prime}}-1} .
$$

Then for the operator $M$ defined in (5.10) with $M_{j j^{\prime}}^{(p)}=\widetilde{M}_{j j^{\prime}}^{(p)}\left(\varepsilon D_{x}\right) \circ g_{p}$, by Assumption $1.2\left(g_{p} \in C_{b}\left([0, \infty) ; H^{s+1}\right) \cap C_{b}^{1}\left([0, \infty) ; H^{s}\right)\right)$, we first have for any $d / 2<\mu \leq s$ and any $u \in H^{\mu}$ that

$$
\|M u\|_{H^{\mu}}+\left\|\left(\partial_{t} M\right) u\right\|_{H^{\mu}} \leq C h_{\varepsilon}^{\alpha-1}\|u\|_{H^{\mu}} .
$$


Moreover, direct computation gives

$$
B_{1}^{t}-i\left[A_{1}\left(\varepsilon D_{x}\right), M\right]-\varepsilon^{2} \partial_{t} M=\widetilde{M}_{r}
$$

with

$$
\widetilde{M}_{r}=-M_{r}^{(1)}-M_{r}^{(2)}+M_{r}^{(3)} .
$$

It is left to show the uniform bound for the operator $\widetilde{M}_{r}$.

Let $d / 2<\mu \leq s$ and $u \in H^{\mu}$. We start estimating $M_{r}^{(1)}$. By Lemma 3.1 and Lemma 3.2, direct computation gives

$$
\begin{aligned}
& \left\|\left[\lambda_{j^{\prime}}, M_{j j^{\prime}}^{(p)}\right] u\right\|_{H^{\mu}}=\left\|\left[\lambda_{j^{\prime}}\left(\varepsilon D_{x}\right), \widetilde{M}_{j j^{\prime}}^{(p)}\left(\varepsilon D_{x}\right) g_{p}\right] u\right\|_{H^{\mu}} \\
& \quad=\left\|\widetilde{M}_{j j^{\prime}}^{(p)}\left(\varepsilon D_{x}\right)\left[\lambda_{j^{\prime}}\left(\varepsilon D_{x}\right), g_{p}\right] u\right\|_{H^{\mu}} \\
& \quad \leq C h_{\varepsilon}^{\alpha-1}\left\|\left[\lambda_{j^{\prime}}\left(\varepsilon D_{x}\right), g_{p}\right] u\right\|_{H^{\mu}} \\
& \quad \leq C \varepsilon h_{\varepsilon}^{\alpha-1}\left\|g_{p}\right\|_{L^{\infty}\left(0, \infty ; H^{s+1}\right)}\|u\|_{H^{\mu}} .
\end{aligned}
$$

Then by the definition of $M_{r}^{(1)}$ in (5.13), we have

$$
\left\|M_{r}^{(1)} u\right\|_{H^{\mu}} \leq C \varepsilon h_{\varepsilon}^{\alpha-1}\|u\|_{H^{\mu}} .
$$

Similarly, for $M_{r}^{(2)}$ and $M_{r}^{(2)}$ defined in (5.14) and (5.15), by Lemma 3.1 and Lemma 3.2, we deduce

$$
\begin{aligned}
& \left\|M_{r}^{(2)} u\right\|_{H^{\mu}} \leq C \varepsilon^{2} h_{\varepsilon}^{\alpha-1}\left\|\partial_{t} g_{p}\right\|_{L^{\infty}\left(0, \infty ; H^{s}\right)}\|u\|_{H^{\mu}}, \\
& \left\|M_{r}^{(3)} u\right\|_{H^{\mu}} \leq C \varepsilon\left\|g_{p}\right\|_{L^{\infty}\left(0, \infty ; H^{s+1}\right)}\|u\|_{H^{\mu}} .
\end{aligned}
$$

Summing up the estimates in (5.20) and (5.21), we obtain

$$
\left\|\widetilde{M}_{r} u\right\|_{H^{\mu}} \leq C\left(\varepsilon h_{\varepsilon}^{\alpha-1}+\varepsilon^{2} h_{\varepsilon}^{\alpha-1}+\varepsilon\right)\|u\|_{H^{\mu}} .
$$

This completes the proof of Proposition 5.2.

\subsection{End of the proof}

In this section, we complete the proof of the first part of Theorem 1.8. This is achieved by choosing $h_{\varepsilon}$ properly. First of all, we choose $h_{\varepsilon}$ such that

$$
\varepsilon^{2} h_{\varepsilon}^{\alpha-1} \rightarrow 0, \text { as } \varepsilon \rightarrow 0 .
$$

By (5.18), we have for any $d / 2<\mu \leq s$ :

$$
\left\|\varepsilon^{2} M\right\|_{\mathcal{L}\left(H^{\mu} \rightarrow H^{\mu}\right)} \rightarrow 0, \text { as } \varepsilon \rightarrow 0 .
$$


Then for $\varepsilon$ sufficient small, the operator $\left(\operatorname{Id}+\varepsilon^{2} M\right)$ is well defined and uniformly bounded from $H^{\mu} \rightarrow H^{\mu}$, and is invertible with a uniformly bounded inverse.

Thus, let $M$ be the operator determined in Proposition 5.2, the change of variable (5.9) is well defined. By Proposition 5.1 and Proposition 5.2, the system (5.11) in $\dot{U}_{2}$ becomes

$$
\partial_{t} \dot{U}_{2}+\frac{i}{\varepsilon^{2}} A_{1}\left(\varepsilon D_{x}\right) \dot{U}_{2}=\left(\varepsilon h_{\varepsilon}^{\alpha-1}+h_{\varepsilon}^{\alpha}+\varepsilon\right) \mathcal{R}_{2},
$$

where there hods the estimate for any $d / 2<\mu \leq s$ :

$$
\left\|\mathcal{R}_{2}(t, \cdot)\right\|_{H^{\mu}} \leq C\left(1+\left\|\dot{U}_{2}(t, \cdot)\right\|_{H^{\mu}}\right)\left\|\dot{U}_{2}(t, \cdot)\right\|_{H^{\mu}} .
$$

Finally $h_{\varepsilon}$ is chosen such that $\varepsilon h_{\varepsilon}^{\alpha-1}=h_{\varepsilon}^{\alpha}$ to achieve the smallest remainder. This is equivalent to

$$
h_{\varepsilon}=\varepsilon,
$$

which implies $\varepsilon h_{\varepsilon}^{\alpha-1}=h_{\varepsilon}^{\alpha}=\varepsilon^{\alpha}$. The condition (5.22) is also satisfied.

For the initial datum of $\dot{U}_{2}$, by (4.9) and (5.9), we have

$$
\dot{U}_{2}(0)=\left(\operatorname{Id}+\varepsilon^{2} M\right)^{-1}\left(\begin{array}{c}
\Pi_{1}\left(\varepsilon D_{x}\right) \psi^{\varepsilon} \\
\vdots \\
\Pi_{3}\left(\varepsilon D_{x}\right) \psi^{\varepsilon}
\end{array}\right)
$$

for which the $H^{s}$ norm is uniformly bounded in $\varepsilon$.

We consider another change of unknown corresponding to a rescalling in time:

$$
\dot{U}_{3}(t)=\dot{U}_{2}\left(\varepsilon^{-\alpha} t\right) \text {. }
$$

Then the equation and initial datum for $\dot{U}_{3}$ are

$$
\left\{\begin{array}{l}
\partial_{t} \dot{U}_{3}+\frac{i}{\varepsilon^{2+\alpha}} A_{1}\left(\varepsilon D_{x}\right) \dot{U}_{3}=\mathcal{R}_{3}, \\
\dot{U}_{3}(0)=\dot{U}_{2}(0)
\end{array}\right.
$$

where $\mathcal{R}_{3}(t):=\left(2+\varepsilon^{1-\alpha}\right) \mathcal{R}_{2}\left(\varepsilon^{-\alpha} t\right)$ satisfies for any $d / 2<\mu \leq s$ :

$$
\left\|\mathcal{R}_{3}(t, \cdot)\right\|_{H^{\mu}} \leq C\left(1+\left\|\dot{U}_{3}(t, \cdot)\right\|_{H^{\mu}}\right)\left\|\dot{U}_{3}(t, \cdot)\right\|_{H^{\mu}} .
$$

Since $s>d / 2$, then by the classical theory for the local-in-time well-posedness of symmetric hyperbolic systems (see for instance Chapter 2 of [20] or Chapter 7 of [22]), there exists a unique local-in-time solution $\dot{U}_{3} \in L^{\infty}\left(0, T_{1} ; H^{s}\right)$ to Cauchy problem (5.26) for some $T_{1}>0$ independent of $\varepsilon$.

Equivalently, there exists a unique solution $\dot{U}_{2} \in L^{\infty}\left(0, \frac{T_{1}}{\varepsilon^{\alpha}} ; H^{s}\right)$ to $(5.23)-(5.25)$. We go back to $\dot{U}$ and obtain the well-posedness of $(4.2)$ in $L^{\infty}\left(0, \frac{T_{1}}{\varepsilon^{\alpha}} ; H^{s}\right)$. Since the approximate solution $U_{a}$ is globally well defined and uniformly bounded in $L^{\infty}\left(0, \infty ; H^{s+1}\right)$, we can reconstruct the solution $U$ for $(1.1)$ in $L^{\infty}\left(0, \frac{T_{1}}{\varepsilon^{\alpha}} ; H^{s}\right)$ through (4.1). We then complete the proof for the first part of Theorem 1.8.

We now turn to prove the second part of Theorem 1.8. 


\section{Long time stability: Part II}

This section is devoted to proving the second part of Theorem 1.8, that is the stability of the approximate solution given in Assumption 1.2 over time $O\left(1 / \varepsilon^{\alpha_{1}}\right)$ with $\alpha_{1}=\min \{2 \alpha, 1\}$. We suppose Assumption 1.1, Assumption 1.2, Assumption 1.6 and Assumption 1.7 are all satisfied.

We will also employ the idea in Section 5. The main idea of singular localization and normal form reduction is the same as in the proof of the first part in Section 5. However, the analysis here is more delicate in order to achieve even longer time stability.

There are two new key points. The first one is to define the singular decomposition $B_{\text {in }}$ and $B_{\text {out }}$, as well as the operator $M$ in the normal form reduction in such a way that we can avoid the commutator $M_{r}^{(1)}$ in (5.13) which is of order $\varepsilon h_{\varepsilon}^{\alpha-1}$ (see (5.19) and (5.20)). This can be achieved by proper choice for the positions of $\chi_{j, j^{\prime}, p}$ in (6.1)-(6.3) and the positions of $g_{p}$ in (6.8) which allows us to force the commutators in (5.13) to appear only associated with the constant eigenmode $\lambda_{j}$ or $\lambda_{j^{\prime}}$ from Assumption 1.7. The other remainders $M_{r}^{(2)}$ and $M_{r}^{(3)}$ are smaller of order $\varepsilon^{2} h_{\varepsilon}^{\alpha-1}$ and $\varepsilon$ respectively (see (5.21)) and we do not need to deal with them furthermore.

The other key point is to avoid the commutators $\left[\chi_{j, j^{\prime}, p}, g_{p}\right]$ which may be large because of the singularity of $\chi_{j, j^{\prime}, p}$ as $\varepsilon \rightarrow 0$. This can be also achieved by choosing the positions of cut-off functions $\chi_{j, j^{\prime}, p},\left(1-\chi_{j, j^{\prime}, p}\right)$ and scalar multiplier $g_{p}$ in the definitions of $B_{\text {in }}, B_{\text {out }}$ and the operator $M$ used in the normal form reduction, see (6.1)-(6.3) and (6.8) later on.

\subsection{Refined singular localization}

The cut-off functions $\chi_{j, j^{\prime}, p}$ are the same as in Section 5.1, while the definitions for the decomposition component $B_{\text {in }}$ and $B_{\text {out }}$ have to be modified.

For any $\left(j, j^{\prime}, p\right)$, we introduce the elements $B_{i n}^{\left(j, j^{\prime}, p\right)}$ and $B_{\text {out }}^{\left(j, j^{\prime}, p\right)}$ in the following way:

- For any $\left(j, j^{\prime}, p\right) \in J_{1}$ which is defined in (5.1), we set

$$
B_{\text {in }}^{\left(j, j^{\prime}, p\right)}:=0, \quad B_{\text {out }}^{\left(j, j^{\prime}, p\right)}:=2 \Pi_{j}\left(\varepsilon D_{x}\right) B\left(U_{0, p}\right) \Pi_{j^{\prime}}\left(\varepsilon D_{x}\right) .
$$

- For any $\left(j, j^{\prime}, p\right) \notin J_{1}$, by Assumption 1.7, one of $\lambda_{j}(\xi)$ and $\lambda_{j^{\prime}}(\xi)$ is constant. If $\lambda_{j}(\xi)$ is constant, we set

$$
\begin{aligned}
B_{i n}^{\left(j, j^{\prime}, p\right)} & :=2 \Pi_{j}\left(\varepsilon D_{x}\right) B\left(U_{0, p}\right) \Pi_{j^{\prime}}\left(\varepsilon D_{x}\right) \chi_{j, j^{\prime}, p}\left(\varepsilon D_{x}\right), \\
B_{\text {out }}^{\left(j, j^{\prime}, p\right)} & :=2 \Pi_{j}\left(\varepsilon D_{x}\right) B\left(U_{0, p}\right) \Pi_{j^{\prime}}\left(\varepsilon D_{x}\right)\left(1-\chi_{j, j^{\prime}, p}\right)\left(\varepsilon D_{x}\right) .
\end{aligned}
$$


If $\lambda_{j^{\prime}}(\xi)$ is constant, we set

$$
\begin{aligned}
B_{i n}^{\left(j, j^{\prime}, p\right)} & :=2 \chi_{j, j^{\prime}, p}\left(\varepsilon D_{x}\right) \Pi_{j}\left(\varepsilon D_{x}\right) B\left(U_{0, p}\right) \Pi_{j^{\prime}}\left(\varepsilon D_{x}\right), \\
B_{\text {out }}^{\left(j, j^{\prime}, p\right)} & :=2\left(1-\chi_{j, j^{\prime}, p}\right)\left(\varepsilon D_{x}\right) \Pi_{j}\left(\varepsilon D_{x}\right) B\left(U_{0, p}\right) \Pi_{j^{\prime}}\left(\varepsilon D_{x}\right) .
\end{aligned}
$$

The new decomposition of $B_{1}:=\widetilde{B}_{\text {in }}+\widetilde{B}_{\text {out }}$ is defined as

$$
\begin{aligned}
& \widetilde{B}_{\text {in }}:=\sum_{p= \pm 1} e^{-i p \omega t / \varepsilon^{2}}\left(B_{\text {in }}^{\left(j, j^{\prime}, p\right)}\right)_{1 \leq j, j^{\prime} \leq J}, \\
& \widetilde{B}_{\text {out }}:=\sum_{p= \pm 1} e^{-i p \omega t / \varepsilon^{2}}\left(B_{\text {out }}^{\left(j, j^{\prime}, p\right)}\right)_{1 \leq j, j^{\prime} \leq J} .
\end{aligned}
$$

We remark that, compared to $B_{\text {in }}$ and $B_{\text {out }}$ defined before in (5.5), the new definition through (6.1)-(6.4) pays more attention to the positions of the cut-offs $\chi_{j, j^{\prime}, p}$. We will see later on in the proof of Proposition 6.2, this choice of positions, together with the choice of positions of $g_{p}$ in (6.8), allows us to avoid relatively large commutators of order $\varepsilon h_{\varepsilon}^{\alpha-1}$ as well as the commutators $\left[\chi_{j, j^{\prime}, p}, g_{p}\right]$.

First of all, similar to Proposition 5.1, we have:

Proposition 6.1. Let $d / 2<\mu \leq s$ and $V \in H^{\mu}$. There holds

$$
\left\|\widetilde{B}_{i n} V\right\|_{H^{\mu}} \leq C\left(h_{\varepsilon}^{\alpha}+\varepsilon\right)\|V\|_{H^{\mu}} .
$$

The proof of Proposition 6.1 is similar as that of Proposition 5.1, that is to employ Lemma 3.1 and Lemma 3.2, Assumption 1.2 and Assumption 1.6, and the property of the cut-off functions $\chi_{j, j^{\prime}, p}$ in (5.2). We do not repeat the details.

\subsection{Refined normal form reduction}

We employ the normal reduction method to deal with $\widetilde{B}_{\text {out }}$ which is localized away from resonance. Introduce the change of variable

$$
\dot{U}_{4}=\left(\mathrm{Id}+\varepsilon^{2} Q\right)^{-1} \dot{U}_{1},
$$

where $U_{1}$ is given in (4.3) and solves (4.8), $Q$ is an operator of the form

$$
Q=\sum_{p= \pm 1} e^{-i p \omega t / \varepsilon^{2}}\left(Q_{j j^{\prime}}^{(p)}\right)_{1 \leq j, j^{\prime} \leq J}
$$

with the operator elements $Q_{j j^{\prime}}^{(p)}$ to be determined. Then, by (4.8), the system in $\dot{U}_{4}$ has the form

$$
\begin{aligned}
\partial_{t} \dot{U}_{4} & +\frac{i}{\varepsilon^{2}} A_{1}\left(\varepsilon D_{x}\right) \dot{U}_{4}=\left(\operatorname{Id}+\varepsilon^{2} Q\right)^{-1}\left(\widetilde{B}_{\text {out }}-i\left[A_{1}\left(\varepsilon D_{x}\right), Q\right]-\varepsilon^{2} \partial_{t} Q\right) \dot{U}_{4} \\
& +\left(\operatorname{Id}+\varepsilon^{2} Q\right)^{-1}\left(\varepsilon^{2} \widetilde{B}_{\text {out }} Q \dot{U}_{4}+\widetilde{B}_{\text {in }}\left(\mathrm{Id}+\varepsilon^{2} Q\right) \dot{U}_{4}+\varepsilon \mathcal{R}_{1}\right) .
\end{aligned}
$$

One key result is the following: 
Proposition 6.2. Let $\widetilde{M}_{j j^{\prime}}^{(p)}(\xi)$ be the symbols defined in (5.16). Then the operator $Q$ defined in (6.6) with

$$
\begin{aligned}
& Q_{j j^{\prime}}^{(p)}:=g_{p} \circ \widetilde{M}_{j j^{\prime}}^{(p)}\left(\varepsilon D_{x}\right) \text { or } \widetilde{M}_{j j^{\prime}}^{(p)}\left(\varepsilon D_{x}\right) \circ g_{p}, \quad \text { if }\left(j, j^{\prime}, p\right) \in J_{1}, \\
& Q_{j j^{\prime}}^{(p)}:=g_{p} \circ \widetilde{M}_{j j^{\prime}}^{(p)}\left(\varepsilon D_{x}\right), \quad \text { if }\left(j, j^{\prime}, p\right) \notin J_{1} \text { and } \lambda_{j}(\cdot) \text { is constant, } \\
& Q_{j j^{\prime}}^{(p)}:=\widetilde{M}_{j j^{\prime}}^{(p)}\left(\varepsilon D_{x}\right) \circ g_{p}, \quad \text { if }\left(j, j^{\prime}, p\right) \notin J_{1} \text { and } \lambda_{j^{\prime}}(\cdot) \text { is constant }
\end{aligned}
$$

satisfies

$$
\widetilde{B}_{\text {out }}-i\left[A_{1}\left(\varepsilon D_{x}\right), Q\right]-\varepsilon^{2} \partial_{t} Q=\left(\varepsilon^{2} h_{\varepsilon}^{\alpha-1}+\varepsilon\right) Q_{r},
$$

where $Q_{r}$ is a linear operator satisfying the estimate:

$$
\left\|Q_{r} V\right\|_{H^{\mu}} \leq C\|V\|_{H^{\mu}}, \quad \text { for any } d / 2<\mu \leq s \text { and } V \in H^{\mu} .
$$

Remark 6.3. We observe that, compared to Proposition 5.2, the remainder estimate in (6.9) is better than that in (5.12) where the term $\varepsilon h_{\varepsilon}^{\alpha-1}$ is eliminated.

Proof of Proposition 6.2. First of all, the operator $Q$ given in Proposition 6.2 is well defined and satisfies for any $d / 2<\mu \leq s$ and $V \in H^{\mu}$ :

$$
\|Q V\|_{H^{\mu}}+\left\|\left(\partial_{t} Q\right) V\right\|_{H^{\mu}} \leq C h_{\varepsilon}^{\alpha-1}\|V\|_{H^{\mu}} .
$$

Given $Q$ of the form (6.6), we compute

$$
\begin{aligned}
& {\left[A_{1}\left(\varepsilon D_{x}\right), Q\right]=A_{1}\left(\varepsilon D_{x}\right) Q-Q A_{1}\left(\varepsilon D_{x}\right)} \\
& =\sum_{p= \pm 1} e^{-i p \omega t / \varepsilon^{2}}\left(\lambda_{j} Q_{j j^{\prime}}^{(p)}-Q_{j j^{\prime}}^{(p)} \lambda_{j^{\prime}}\right)_{1 \leq j, j^{\prime} \leq J},
\end{aligned}
$$

where we used the simplified notation $\lambda_{j}:=\lambda_{j}\left(\varepsilon D_{x}\right), j \in\{1, \cdots, J\}$.

We then compute

$$
\varepsilon^{2} \partial_{t} Q=\sum_{p= \pm 1} e^{-i p \omega t / \varepsilon^{2}}(-i p \omega)\left(Q_{j j^{\prime}}^{(p)}\right)_{1 \leq j, j^{\prime} \leq J}+Q_{r, 1}
$$

where

$$
Q_{r, 1}:=\varepsilon^{2} \sum_{p= \pm 1} e^{-i p \omega t / \varepsilon^{2}}\left(\partial_{t} Q_{j j^{\prime}}^{(p)}\right)_{1 \leq j, j^{\prime} \leq J}
$$

Direct computation gives that for any $d / 2<\mu \leq s$ and $V \in H^{\mu}$ :

$$
\left\|Q_{r, 1} V\right\|_{H^{\mu}} \leq C \varepsilon^{2} h_{\varepsilon}^{\alpha-1}\|V\|_{H^{\mu}} .
$$

Then

$$
\widetilde{B}_{\text {out }}-i\left[A_{1}\left(\varepsilon D_{x}\right), Q\right]-\varepsilon^{2} \partial_{t} Q=\sum_{p= \pm 1} e^{-i p \omega t / \varepsilon^{2}}\left(Q_{j, j^{\prime}, p}^{(r)}\right)_{1 \leq j, j^{\prime} \leq J}-Q_{r, 1},
$$

where

$$
Q_{j, j^{\prime}, p}^{(r)}:=B_{\text {out }}^{\left(j, j^{\prime}, p\right)}-i\left(\lambda_{j} Q_{j j^{\prime}}^{(p)}-Q_{j j^{\prime}}^{(p)} \lambda_{j^{\prime}}-p \omega Q_{j j^{\prime}}^{(p)}\right) .
$$

We now estimate $Q_{j, j^{\prime}, p}^{(r)}$ case by case: 
- For $\left(j, j^{\prime}, p\right) \in J_{1}$, we choose $Q_{j j^{\prime}}^{(p)}=g_{p} \circ \widetilde{M}_{j j^{\prime}}^{(p)}\left(\varepsilon D_{x}\right)$ (the same result follows if we choose $\left.Q_{j j^{\prime}}^{(p)}=\widetilde{M}_{j j^{\prime}}^{(p)}\left(\varepsilon D_{x}\right) \circ g_{p}\right)$, then

$$
\begin{aligned}
Q_{j, j^{\prime}, p}^{(r)} & =2 \Pi_{j}\left(\varepsilon D_{x}\right) B\left(g_{p} e_{p}\right) \Pi_{j^{\prime}}\left(\varepsilon D_{x}\right)-2 g_{p} \Pi_{j}\left(\varepsilon D_{x}\right) B\left(e_{p}\right) \Pi_{j^{\prime}}\left(\varepsilon D_{x}\right)+Q_{r, 2}^{j, j^{\prime}, p} \\
& =Q_{r, 2}^{j, j^{\prime}, p}+Q_{r, 3}^{j, j^{\prime}, p}
\end{aligned}
$$

where

$$
Q_{r, 2}^{j, j^{\prime}, p}:=-2\left[\lambda_{j}, g_{p}\right] \widetilde{M}_{j j^{\prime}}^{(p)}\left(\varepsilon D_{x}\right), \quad Q_{r, 3}^{j, j^{\prime}, p}:=2\left[\Pi_{j}\left(\varepsilon D_{x}\right), g_{p}\right] B\left(e_{p}\right) \Pi_{j^{\prime}}\left(\varepsilon D_{x}\right) .
$$

For any $\left(j, j^{\prime}, p\right) \in J_{1}, \alpha_{j, j^{\prime}, p}=1$. Then by (5.17) and the estimates in Lemma 3.1 and (3.2), we have for any $d / 2<\mu \leq s$ and $u \in H^{\mu}$ :

$$
\left\|Q_{r, 2}^{j, j^{\prime}, p} u\right\|_{H^{\mu}}+\left\|Q_{r, 3}^{j, j^{\prime}, p} u\right\|_{H^{\mu}} \leq C \varepsilon\|u\|_{H^{\mu}} .
$$

- For $\left(j, j^{\prime}, p\right) \notin J_{1}$ with $\lambda_{j}(\cdot)$ constant, we have

$$
\begin{aligned}
Q_{j, j^{\prime}, p}^{(r)}= & 2 \Pi_{j}\left(\varepsilon D_{x}\right) B\left(g_{p} e_{p}\right) \Pi_{j^{\prime}}\left(\varepsilon D_{x}\right)\left(1-\chi_{j, j^{\prime}, p}\right)\left(\varepsilon D_{x}\right) \\
& -2 g_{p} \Pi_{j}\left(\varepsilon D_{x}\right) B\left(e_{p}\right) \Pi_{j^{\prime}}\left(\varepsilon D_{x}\right)\left(1-\chi_{j, j^{\prime}, p}\right)\left(\varepsilon D_{x}\right)+Q_{r, 4}^{j, j^{\prime}, p} \\
= & Q_{r, 4}^{j, j^{\prime}, p}+Q_{r, 5}^{j, j^{\prime}, p},
\end{aligned}
$$

where

$$
\begin{aligned}
& Q_{r, 4}^{j, j^{\prime}, p}:=-2\left[\lambda_{j}, g_{p}\right] \widetilde{M}_{j j^{\prime}}^{(p)}\left(\varepsilon D_{x}\right)=0 \\
& Q_{r, 5}^{j, j^{\prime}, p}:=2\left[\Pi_{j}\left(\varepsilon D_{x}\right), g_{p}\right] B\left(e_{p}\right) \Pi_{j^{\prime}}\left(\varepsilon D_{x}\right)\left(1-\chi_{j, j^{\prime}, p}\right)\left(\varepsilon D_{x}\right),
\end{aligned}
$$

where we used the fact $\lambda_{j}$ is constant. Again by the estimates in Lemma 3.1 and Lemma 3.2, we have for any $d / 2<\mu \leq s$ and $u \in H^{\mu}$ :

$$
\left\|Q_{r, 5}^{j, j^{\prime}, p} u\right\|_{H^{\mu}} \leq C \varepsilon\|u\|_{H^{\mu}} .
$$

- For $\left(j, j^{\prime}, p\right) \notin J_{1}$ with $\lambda_{j^{\prime}}(\cdot)$ constant, we have

$$
\begin{aligned}
Q_{j, j^{\prime}, p}^{(r)}= & 2\left(1-\chi_{j, j^{\prime}, p}\right)\left(\varepsilon D_{x}\right) \Pi_{j}\left(\varepsilon D_{x}\right) B\left(g_{p} e_{p}\right) \Pi_{j^{\prime}}\left(\varepsilon D_{x}\right) \\
& -2\left(1-\chi_{j, j^{\prime}, p}\right)\left(\varepsilon D_{x}\right) \Pi_{j}\left(\varepsilon D_{x}\right) B\left(e_{p}\right) \Pi_{j^{\prime}}\left(\varepsilon D_{x}\right) g_{p}+Q_{r, 6}^{j, j^{\prime}, p} \\
= & Q_{r, 6}^{j, j^{\prime}, p}+Q_{r, 7}^{j, j^{\prime}, p},
\end{aligned}
$$

where

$$
\begin{aligned}
& Q_{r, 6}^{j, j^{\prime}, p}:=-2 \widetilde{M}_{j j^{\prime}}^{(p)}\left(\varepsilon D_{x}\right)\left[\lambda_{j^{\prime}}, g_{p}\right]=0 \\
& Q_{r, 7}^{j, j^{\prime}, p}:=2\left(1-\chi_{j, j^{\prime}, p}\right)\left(\varepsilon D_{x}\right) \Pi_{j}\left(\varepsilon D_{x}\right) B\left(e_{p}\right)\left[g_{p}, \Pi_{j^{\prime}}\left(\varepsilon D_{x}\right)\right],
\end{aligned}
$$

where we used the fact $\lambda_{j^{\prime}}$ is constant. Moreover, for any $d / 2<\mu \leq s$ and $u \in H^{\mu}$ there holds

$$
\left\|Q_{r, 7}^{j, j^{\prime}, p} u\right\|_{H^{\mu}} \leq C \varepsilon\|u\|_{H^{\mu}} .
$$

By the estimates in (6.11), (6.12), (6.13) and (6.14), we conclude our result in Proposition 6.2. 


\subsection{End of the proof}

In this section, we complete the proof of the first part of Theorem 1.8. Let $h_{\varepsilon}$ be such that

$$
\varepsilon^{2} h_{\varepsilon}^{\alpha-1} \rightarrow 0, \text { as } \varepsilon \rightarrow 0 .
$$

Then by (6.10) for $\varepsilon$ sufficient small, the operator $\left(\operatorname{Id}+\varepsilon^{2} Q\right)$ as well as its inverse are uniformly bounded from $H^{\mu} \rightarrow H^{\mu}$. Thus, the change of variable (6.5) is well defined. By Proposition 6.1 and Proposition 6.2, the system (6.7) in $\dot{U}_{4}$ becomes

$$
\partial_{t} \dot{U}_{4}+\frac{i}{\varepsilon^{2}} A_{1}\left(\varepsilon D_{x}\right) \dot{U}_{4}=\left(\varepsilon^{2} h_{\varepsilon}^{\alpha-1}+h_{\varepsilon}^{\alpha}+\varepsilon\right) \mathcal{R}_{4},
$$

where there holds the estimate for any $d / 2<\mu \leq s$ :

$$
\left\|\mathcal{R}_{4}(t, \cdot)\right\|_{H^{\mu}} \leq C\left(1+\left\|\dot{U}_{4}(t, \cdot)\right\|_{H^{\mu}}\right)\left\|\dot{U}_{4}(t, \cdot)\right\|_{H^{\mu}} .
$$

Finally $h_{\varepsilon}$ is chosen such that $\varepsilon^{2} h_{\varepsilon}^{\alpha-1}=h_{\varepsilon}^{\alpha}$ to achieve the smallest remainder. This suggests

$$
h_{\varepsilon}=\varepsilon^{2} .
$$

This implies $\varepsilon^{2} h_{\varepsilon}^{\alpha-1}=h_{\varepsilon}^{\alpha}=\varepsilon^{2 \alpha}$. The condition (6.15) is also satisfied.

For the initial datum of $\dot{U}_{4}$, by (4.9) and (5.9), we have

$$
\dot{U}_{4}(0)=\left(\operatorname{Id}+\varepsilon^{2} Q\right)^{-1}\left(\begin{array}{c}
\Pi_{1}\left(\varepsilon D_{x}\right) \psi^{\varepsilon} \\
\vdots \\
\Pi_{3}\left(\varepsilon D_{x}\right) \psi^{\varepsilon}
\end{array}\right)
$$

for which the $H^{s}$ norm is uniformly bounded in $\varepsilon$.

We consider another change of unknown corresponding to a rescalling in time:

$$
\dot{U}_{5}(t)=\dot{U}_{4}\left(\varepsilon^{-\alpha_{1}} t\right), \quad \alpha_{1}:=\min \{2 \alpha, 1\} .
$$

Then the equation and initial datum for $\dot{U}_{5}$ are

$$
\left\{\begin{array}{l}
\partial_{t} \dot{U}_{5}+\frac{i}{\varepsilon^{2+\alpha_{1}}} A_{1}\left(\varepsilon D_{x}\right) \dot{U}_{5}=\mathcal{R}_{5}, \\
\dot{U}_{5}(0)=\dot{U}_{4}(0)
\end{array}\right.
$$

where $\mathcal{R}_{5}(t):=\left(2 \varepsilon^{2 \alpha-\alpha_{1}}+\varepsilon^{1-\alpha_{1}}\right) \mathcal{R}_{4}\left(\varepsilon^{-\alpha_{1}} t\right)$ satisfies for any $d / 2<\mu \leq s$ :

$$
\left\|\mathcal{R}_{5}(t, \cdot)\right\|_{H^{\mu}} \leq C\left(1+\left\|\dot{U}_{5}(t, \cdot)\right\|_{H^{\mu}}\right)\left\|\dot{U}_{5}(t, \cdot)\right\|_{H^{\mu}} .
$$

Then the classical theory gives the local-in-time existence and uniqueness of the solution $\dot{U}_{5} \in L^{\infty}\left(0, T_{2} ; H^{s}\right)$ to Cauchy problem (6.17) for some $T_{2}>0$ independent 
of $\varepsilon$. Going back to $\dot{U}$ gives the well-posedness of $(4.2)$ in $L^{\infty}\left(0, \frac{T_{2}}{\varepsilon^{\alpha_{1}}} ; H^{s}\right)$. Since the approximate solution $U_{a}$ is globally well defined and uniformly bounded in $L^{\infty}\left(0, \infty ; H^{s+1}\right)$, we can reconstruct the solution $U$ for $(1.1)$ in $L^{\infty}\left(0, \frac{T_{2}}{\varepsilon^{\alpha_{1}}} ; H^{s}\right)$ through (4.1). We then complete the proof for the second part of Theorem 1.8.

Now we have finished the proof of Theorem 1.8. In the next section, we apply our result to the study of non-relativistic limit problems of Klein-Gordon equations.

\section{Example and application}

Our example contains the non-relativistic limit problems of Klein-Gordon equations. The Klein-Gordon equation is a relativistic version of the Schrödinger equation and is used to describe the motion of a spinless particle with positive mass $m>0$. Let $c$ be the speed of light, $h$ be the Planck constant, then the typical form of the Klein-Gordon equation is

$$
\frac{h^{2}}{m c^{2}} \partial_{t t} u-\frac{h^{2}}{m} \Delta u+m c^{2} u=f(u), \quad t \geq 0, \quad x \in \mathbb{R}^{d} .
$$

Here $u=u(t, x)$ is a real-valued (or complex-valued) field, and $f(u)$ is a realvalued (or complex-valued) function. By normalizing the mass such that $m=1$ and rescaling the time and space variables as

$$
\tilde{u}(t, x):=u\left(h^{-1} t, h^{-1} x\right)
$$

and by introducing $\varepsilon=c^{-1}$, we arrive at the following non-dimensional form of the Klein-Gordon equation

$$
\varepsilon^{2} \partial_{t t} u-\Delta u+\frac{1}{\varepsilon^{2}} u=f(u), \quad t \geq 0, \quad x \in \mathbb{R}^{d} .
$$

Here in (7.1), we denote the new unknown $\tilde{u}$ still by the original notation $u$.

For fixed $\varepsilon$, the well-posedness of the Klein-Gordon equation is well studied (see for instance $[8,9]$ ). Our concern is the long time asymptotic behavior of the solution in the non-relativistic limit $(\varepsilon \rightarrow 0)$ with real initial data of the form

$$
u(0)=u_{0, \varepsilon}, \quad\left(\partial_{t} u\right)(0)=\frac{1}{\varepsilon^{2}} u_{1, \varepsilon}
$$

The local-in-time asymptotic behavior in the non-relativistic limit of (7.1)-(7.2) is well studied both in mathematical analysis and in numerical computations, see for instance $[21,1]$ and the recent result concerning higher order approximation by the authors in [19]. However, concerning the long time (for example of order $O(1 / \varepsilon)$ ) asymptotic behavior in this setting, there are few results according to the authors' knowledge. 


\subsection{Setting and main result}

With quadratic nonlinearity $f(u)=\lambda u^{2}, \lambda \in \mathbb{R}$, we will show that up to a change of unknowns, the Klein-Gordon equation (7.1) can be treated as a system of the form (1.1). With additional regularity assumption on the initial data in (7.2), we verify that Assumption 1.1, Assumption 1.2, Assumption 1.6 and Assumption 1.7 are all satisfied. Hence, we can apply Theorem 1.8 to obtain long time $O(1 / \varepsilon)$ stability property. Moreover, the leading term of the approximate solution solves linear Schrödinger equation. This shows rigourously that over long time of order $O(1 / \varepsilon)$, the quadratic Klein-Gordon equation can be well approximated by the linear Schrödinger equation in the non-relativistic regime $\varepsilon \rightarrow 0$. However, this example model is rather non-physical since physical nonlinearities are of the form $f(u)=$ $g\left(|u|^{2}\right) u$ which fulfills the gauge invariance. An extension of the theory presented in this paper to non quadratic nonlinearities is needed to consider such physical nonlinearities.

We state our result.

Theorem 7.1. Assume that the real initial datum $\left(u_{0, \varepsilon}, u_{1, \varepsilon}\right)$ has the form

$$
u_{0, \varepsilon}=\varphi_{0}+\varepsilon \varphi_{\varepsilon}, \quad u_{1, \varepsilon}=\psi_{0}+\varepsilon \psi_{\varepsilon}
$$

with

$$
\begin{aligned}
& \left(\varphi_{0}, \psi_{0}\right) \in\left(H^{s}\right)^{2} \quad \text { independent of } \varepsilon \\
& \left\{\left(\varphi_{\varepsilon}, \psi_{\varepsilon}, \varepsilon \nabla \varphi_{\varepsilon}\right)\right\}_{0<\varepsilon<1} \text { uniformly bounded in }\left(H^{s-4}\right)^{d+2}
\end{aligned}
$$

for some $s>d / 2+4$. Then there exists $\varepsilon_{0}>0$ such that for any $0<\varepsilon<\varepsilon_{0}$ the Cauchy problem (7.1)-(7.2) with $f(u)=\lambda u^{2}, \lambda \in \mathbb{R}$ admits a unique solution $u \in L^{\infty}\left(0, \frac{T}{\varepsilon} ; H^{s-4}\right)$ for some $T>0$ independent of $\varepsilon$. Moreover, there exists a constant $C$ independent of $\varepsilon$ such that

$$
\left\|u-\left(e^{-i t / \varepsilon^{2}} v+e^{i t / \varepsilon^{2}} \bar{v}\right)\right\|_{L^{\infty}\left(0, \frac{T}{\varepsilon} ; H^{s-4}\right)} \leq C \varepsilon
$$

where $v \in C_{b}\left(0, \infty ; H^{s}\right) \cap C_{b}^{1}\left(0, \infty ; H^{s-2}\right)$ is the solution to the following Cauchy problem associate with the linear Schrödinger equation

$$
2 i v_{t}+\Delta v=0, \quad v(0)=\frac{\varphi_{0}+i \psi_{0}}{2} .
$$

The rest of this section is devoted to the proof of Theorem 7.1.

\subsection{Reformulation of the equation}

We rewrite the Klein-Gordon equation (7.1) as a symmetric hyperbolic system by introducing

$$
U:=(w, v, u):=\left(\varepsilon \nabla^{T} u, \varepsilon^{2} \partial_{t} u, u\right)^{T}:=\left(\varepsilon\left(\partial_{x_{1}} u, \cdots, \partial_{x_{d}} u\right), \varepsilon^{2} \partial_{t} u, u\right)^{T} .
$$


Here the notation $\nabla:=\left(\partial_{x_{1}}, \cdots, \partial_{x_{d}}\right)^{T}$ is of column form. We remark that 0 could be the scalar number zero, the zero column vector $0_{d}$, the zero row vector $0_{d}^{T}$ or the zero matrix $0_{d \times d}$, but we will not specify if there is no confusion in the context.

Then the equation (7.1) is equivalent to

$$
\partial_{t} U+\frac{1}{\varepsilon} A\left(\partial_{x}\right) U+\frac{1}{\varepsilon^{2}} A_{0} U=F(U),
$$

where

$$
A\left(\partial_{x}\right):=-\left(\begin{array}{ccc}
0 & \nabla & 0 \\
\nabla^{T} & 0 & 0 \\
0 & 0 & 0
\end{array}\right), \quad A_{0}:=\left(\begin{array}{ccc}
0 & 0 & 0 \\
0 & 0 & 1 \\
0 & -1 & 0
\end{array}\right), \quad F(U)=\left(\begin{array}{c}
0 \\
f(u) \\
0
\end{array}\right) .
$$

We consider in this paper the quadratic nonlinearity of the form $f(u)=\lambda u^{2}$ for some $\lambda>0$, we can write

$$
F(U)=B(U, U)
$$

with $B$ a symmetric bilinear form defined as

$$
B\left(U_{1}, U_{2}\right)=-\lambda\left(\begin{array}{c}
0 \\
u_{1} u_{2} \\
0
\end{array}\right), \quad \text { for any } U_{j}=\left(\begin{array}{c}
w_{j} \\
v_{j} \\
u_{j}
\end{array}\right), j \in\{1,2\} .
$$

Moreover, by (7.2) and the assumption in Theorem 7.1, the initial datum is

$$
U(0)=\left(\varepsilon \nabla^{T} u_{0, \varepsilon}, u_{1, \varepsilon}, u_{0, \varepsilon}\right)^{T}=\left(0, \psi_{0}, \phi_{0}\right)^{T}+\varepsilon\left(\nabla^{T}\left(\phi_{0}+\phi_{\varepsilon}\right), \psi_{\varepsilon}, \phi_{\varepsilon}\right)^{T} .
$$

Thus, we obtain a Cauchy problem (7.5)-(7.9) which has the form of (1.1).

\subsection{Spectral decomposition}

We rewrite the linear differential operator on the left-hand side of (7.5) as

$$
\partial_{t}+\frac{i}{\varepsilon^{2}}\left(A\left(\varepsilon D_{x}\right)+A_{0} / i\right), \quad D_{x}:=\partial_{x} / i
$$

The symbol of the semiclassical Fourier multiplier $\left(A\left(\varepsilon D_{x}\right)+A_{0} / i\right)$ is

$$
A(\xi)+A_{0} / i
$$

which is a symmetric matrix for any $\xi \in \mathbb{R}^{d}$. Direct computation gives the following smooth spectral decomposition

$$
A(\xi)+A_{0} / i=\lambda_{1}(\xi) \Pi_{1}(\xi)+\lambda_{2}(\xi) \Pi_{2}(\xi)+\lambda_{3}(\xi) \Pi_{3}(\xi)
$$


with the eigenvalues

$$
\lambda_{1}(\xi)=\sqrt{1+|\xi|^{2}}=\langle\xi\rangle, \quad \lambda_{2}(\xi)=-\sqrt{1+|\xi|^{2}}=-\langle\xi\rangle, \quad \lambda_{3}(\xi) \equiv 0
$$

and eigenprojections

$$
\Pi_{j}(\xi)=\frac{1}{2}\left(\begin{array}{ccc}
\frac{\xi \xi^{T}}{\lambda_{j}^{2}} & \frac{\xi}{\lambda_{j}} & \frac{-i \xi}{\lambda_{j}^{2}} \\
\frac{\xi^{T}}{\lambda_{j}} & 1 & \frac{-i}{\lambda_{j}} \\
\frac{i \xi^{T}}{\lambda_{j}^{2}} & \frac{i}{\lambda_{j}} & \frac{1}{\lambda_{j}^{2}}
\end{array}\right), \quad \Pi_{3}(\xi)=\frac{1}{d+|\xi|^{2}}\left(\begin{array}{ccc}
\operatorname{Id}_{d} & 0 & -i \xi \\
0 & 0 & 0 \\
i \xi^{T} & 0 & |\xi|^{2}
\end{array}\right)
$$

where $j \in\{1,2\}, \xi=\left(\xi_{1}, \cdots, \xi_{d}\right)^{T}$ is a column vector and $\operatorname{Id}_{d}$ denotes the unit matrix of order $d$. It is direct to check that $\lambda_{j} \in S^{1}$ and $\Pi_{j} \in S^{0}$ for any $j \in\{1,2,3\}$. Clearly, we have $\lambda_{j} \in S^{1}, \Pi_{j} \in S^{0}, j \in\{1,2,3\}$. As a result, Assumption 1.1 is satisfied.

According to (7.10), we can write

$$
A\left(\varepsilon D_{x}\right)+A_{0} / i=\lambda_{1}\left(\varepsilon D_{x}\right) \Pi_{1}\left(\varepsilon D_{x}\right)+\lambda_{2}\left(\varepsilon D_{x}\right) \Pi_{2}\left(\varepsilon D_{x}\right) .
$$

\subsection{WKB approximate solution}

In this section, we use WKB expansion to construct an approximate solution to Cauchy problem (7.5)-(7.9). Moreover, we will show that this approximate solution is global-in-time well defined and uniformly bounded. As a result, Assumption 1.2 is verified.

\subsubsection{WKB cascade}

We make a formal power series expansion in $\varepsilon$ for the solution and each term in the series is a trigonometric polynomial in $\theta:=-t / \varepsilon^{2}$ :

$$
U_{a}=\sum_{n=0}^{K_{a}+1} \varepsilon^{n} U_{n}, \quad U_{n}=\sum_{p \in \mathbb{Z}} e^{i p \theta} U_{n, p}, \quad K_{a} \in \mathbb{Z}_{+} .
$$

The amplitudes $U_{n, p}(t, x)$ are not highly-oscillating (independent of $\theta$ ) and satisfy $U_{n,-p}=\bar{U}_{n, p}$ due to the reality of $U_{a}$. We plug (7.13) into (7.5) and deduce the system of order $O\left(\varepsilon^{n}\right), n=-2,-1,0,1$.

We start from considering the equations in the terms of order $O\left(\varepsilon^{-2}\right)$. We reproduce such equations as follows

$$
\left(-i p+A_{0}\right) U_{0, p}=0, \quad \text { for all } p .
$$

It is easy to find that $\left(-i p+A_{0}\right)$ are invertible except when $p \in \mathcal{H}_{0}:=\{-1,0,1\}$. We then deduce from (7.14) that

$$
U_{0, p}=0, \quad \text { for all } p \text { such that }|p| \geq 2 .
$$


We do not need to include the mean mode $U_{0,0}$ in the approximation. For simplicity, we take

$$
U_{0,0}=0
$$

For $p=1,(7.14)$ is equivalent to the so called polarization condition $U_{0, p} \in$ $\operatorname{ker}(i p+A)$. This implies

$$
U_{0,1}=g_{0} e_{1}, \quad e_{1}:=\left(0_{d}^{T},-i, 1\right)^{T}, \quad g_{0} \text { is a scalar function. }
$$

For $p=-1$, reality implies

$$
U_{0,-1}=\bar{U}_{0,1}=\bar{g}_{0} e_{-1}, \quad e_{-1}:=\bar{e}_{1}=\left(0_{d}^{T}, i, 1\right)^{T} .
$$

We continue to consider the equations in the terms of order $O\left(\varepsilon^{-1}\right)$ :

$$
A\left(\partial_{x}\right) U_{0, p}+\left(-i p+A_{0}\right) U_{1, p}=0, \text { for all } p \text {. }
$$

When $p=0$, by the choice of the leading mean mode in (7.16), equation (7.19) becomes

$$
A_{0} U_{1,0}=0
$$

which is equivalent to

$$
U_{1,0}=\left(h_{1}^{T}, 0,0\right)^{T} \quad \text { for some vector valued function } h_{1} \in \mathbb{R}^{d} .
$$

When $p=1$, by (7.17), equation (7.19) is equivalent to

$$
U_{1,1}=g_{1} e_{1}+\left(\nabla^{T} g_{0}, 0,0\right)^{T} \text { for some scalar function } g_{1} \text {. }
$$

When $|p| \geq 2$, the invertibility of $\left(-i p+A_{0}\right)$ and (7.15) imply

$$
U_{1, p}=0, \quad \text { for all } p \text { such that }|p| \geq 2 \text {. }
$$

The equations in the terms of order $O\left(\varepsilon^{0}\right)$ are as follows:

$$
\partial_{t} U_{0, p}+A\left(\partial_{x}\right) U_{1, p}+\left(-i p+A_{0}\right) U_{2, p}=\sum_{p_{1}+p_{2}=p} B\left(U_{0, p_{1}}, U_{0, p_{2}}\right), \quad \text { for all } p .
$$

When $p=0$, by (7.8), (7.15)-(7.18), equation (7.23) becomes

$$
A\left(\partial_{x}\right) U_{1,0}+A_{0} U_{2,0}=2 B\left(U_{0,1}, U_{0,-1}\right)=-2 \lambda\left(0_{d}^{T},\left|g_{0}\right|^{2}, 0\right)^{T}
$$

which is equivalent to (by employing (7.6) and (7.20))

(7.24) $U_{2,0}=\left(h_{2}^{T}, 0, \operatorname{div} h_{1}-2 \lambda\left|g_{0}\right|^{2}\right)^{T} \quad$ for some vector valued function $h_{2} \in \mathbb{R}^{d}$. 
When $p=1$, by (7.6), (7.8), (7.15) and (7.16), equation (7.23) becomes

$$
\partial_{t} U_{0,1}+A\left(\partial_{x}\right) U_{1,1}+\left(-i+A_{0}\right) U_{2,1}=0
$$

By (7.17) and (7.21), equation (7.25) is equivalent to

$$
\left\{\begin{array}{l}
2 i \partial_{t} g_{0}+\Delta g_{0}=0, \\
U_{2,1}=g_{2} e_{1}+\left(\nabla^{T} g_{1}, \partial_{t} g_{0}, 0\right)^{T}, \quad \text { for some scalar function } g_{2} .
\end{array}\right.
$$

This is how we obtain the linear Schrödinger equation (7.4). The initial datum of $g_{0}$ is determined in such a way that $U_{0}(0)=\left(0_{d}^{T}, \psi_{0}, \varphi_{0}\right)^{T}$ which is the leading term of initial data $U(0)$ (see (7.9)). This imposes

$$
g_{0}(0)=\frac{\varphi_{0}+i \psi_{0}}{2}
$$

When $p=2$, by (7.6), (7.8), (7.15)-(7.17), (7.22), equation (7.23) becomes

$$
\left(-2 i+A_{0}\right) U_{2,2}=B\left(U_{0,1}, U_{0,1}\right)=-2 \lambda\left(0_{d}^{T}, g_{0}^{2}, 0\right)^{T}
$$

which is equivalent to

$$
U_{2,2}=\frac{\lambda}{3}\left(0_{d}^{T},-2 i g_{0}^{2}, g_{0}^{2}\right)^{T}
$$

When $|p| \geq 3$, equation (7.23) implies

$$
U_{2, p}=0, \quad \text { for all } p \text { such that }|p| \geq 3 .
$$

We finally consider the equations of order $O(\varepsilon)$ :

$$
\partial_{t} U_{1, p}+A\left(\partial_{x}\right) U_{2, p}+\left(-i p+A_{0}\right) U_{3, p}=2 \sum_{p_{1}+p_{2}=p} B\left(U_{0, p_{1}}, U_{1, p_{2}}\right), \quad \text { for all } p .
$$

When $p=0$, by (7.6), (7.8), (7.15)-(7.18), (7.21), (7.22), equation (7.29) becomes

$$
\partial_{t} U_{1,0}+A\left(\partial_{x}\right) U_{2,0}+A_{0} U_{3,0}=4 \Re B\left(U_{0,1}, U_{1,-1}\right)=-4 \lambda\left(0_{d}^{T}, \Re\left(g_{0} \bar{g}_{1}\right), 0\right)^{T}
$$

which is equivalent to (by (7.20) and (7.24))

$$
\partial_{t} h_{1}=0, U_{3,0}=\left(h_{3}^{T}, 0, \operatorname{div} h_{2}-4 \lambda \Re\left(g_{0} \bar{g}_{1}\right)\right)^{T},
$$

for some vector valued function $h_{3} \in \mathbb{R}^{d}$. The notation $\Re a$ stands for the real part of $a$. 
Here we take a trivial solution $h_{1}=0$ to the equation $\partial_{t} h_{1}=0$ in (7.30). By (7.20), this means

$$
U_{1,0}=0
$$

When $p=1$, by (7.8), (7.15), (7.16), (7.22) and (7.31), equation (7.29) becomes

$$
\partial_{t} U_{1,1}+A\left(\partial_{x}\right) U_{2,1}+\left(-i+A_{0}\right) U_{3,1}=0
$$

which is equivalent to

$$
\left\{\begin{array}{l}
2 i \partial_{t} g_{1}+\Delta g_{1}=0 \\
U_{3,1}=g_{3} e_{1}+\left(\nabla^{T} g_{2}, \partial_{t} g_{1}, 0\right)^{T}, \text { for some scalar function } g_{3} .
\end{array}\right.
$$

Here we used (7.21) and (7.26).

We find that $g_{1}$ satisfies the same linear Schrödinger equation as $g_{0}$. Since we do not need to include initial data of $g_{1}$ (this may be needed sometimes in order to have a better initial approximation), we will take a trivial solution $g_{1}=0$.

When $p=2$, by (7.6), (7.8), (7.17), (7.15), (7.21), (7.22) and (7.31), equation (7.29) becomes

$$
A\left(\partial_{x}\right) U_{2,2}+\left(-2 i+A_{0}\right) U_{3,2}=2 B\left(U_{0,1}, U_{1,1}\right)=-2 \lambda\left(0_{d}^{T}, g_{0} g_{1}, 0\right)^{T}
$$

which is equivalent to (by (7.28))

$$
U_{3,2}=\frac{2 \lambda}{3}\left(g_{0} \nabla^{T} g_{0},-2 i g_{0} g_{1}, g_{0} g_{1}\right)^{T} .
$$

When $|p| \geq 3,(7.29)$ is equivalent to

$$
U_{3, p}=0, \quad \text { for all } p \text { such that }|p| \geq 3 \text {. }
$$

\subsubsection{WKB approximate solution}

By (7.3), we have $g_{0}(0) \in H^{s}$ with $s>d / 2+4$. Then classically there exists a unique global-in-time solution $g_{0}$ to the Cauchy problem $(7.26)_{1}-(7.27)$ in Sobolev space $H^{s}$. Moreover, we have the estimate

$$
\left\|\partial_{t} g_{0}\right\|_{L^{\infty}\left(0, \infty ; H^{s-2}\right)} \leq C\left\|g_{0}\right\|_{L^{\infty}\left(0, \infty ; H^{s}\right)} \leq C\left\|\left(\phi_{0}, \psi_{0}\right)\right\|_{H^{s}}
$$

To construct an approximate solution, we need to determine $g_{j}$ and $h_{j}, j \in\{1,2,3\}$, appeared in Section 7.4.1. Taking

$$
g_{1}=g_{2}=g_{3}=h_{1}=h_{2}=h_{3}=0
$$


implies, by employing the argument in Section 7.4.1, that

$$
\begin{aligned}
& U_{0,1}=g_{0} e_{1}, \quad U_{1,1}=\left(\begin{array}{c}
\nabla g_{0} \\
0 \\
0
\end{array}\right), \quad U_{2,0}=-2 \lambda\left(\begin{array}{c}
0_{d} \\
0 \\
\left|g_{0}\right|^{2}
\end{array}\right), \\
& U_{2,1}=\left(\begin{array}{c}
0_{d} \\
\partial_{t} g_{0} \\
0
\end{array}\right), \quad U_{2,2}=\frac{\lambda}{3}\left(\begin{array}{c}
0_{d} \\
-2 i g_{0}^{2} \\
g_{0}^{2}
\end{array}\right), \quad U_{3,2}=\left(\begin{array}{c}
g_{0} \nabla g_{0} \\
0 \\
0
\end{array}\right),
\end{aligned}
$$

and $U_{n, p}=0$ for all other $(n, p) \in \mathbb{Z}^{2}, p \geq 0$, and $U_{n, p}=\bar{U}_{n,-p}$ for $p<0$.

We observe that all the components in (7.33) are determined by the leading amplitude $g_{0}$. By the estimate of $g_{0}$ in $(7.32)$, we have for any $(n, p) \in \mathbb{Z}^{2}$ :

$$
U_{n, p} \in L^{\infty}\left(0, \infty ; H^{s-2}\right), \quad \partial_{t} U_{n, p} \in L^{\infty}\left(0, \infty ; H^{s-4}\right) .
$$

Plugging all such $U_{n, p}$ into (7.13) gives an approximate solution $U_{a}$ of the form

$$
U_{a}=U_{0}+\varepsilon U_{1}+\varepsilon^{2} U_{2}+\varepsilon^{3} U_{3}
$$

which solves the following Cauchy problem globally in time

$$
\left\{\begin{array}{l}
\partial_{t} U_{a}+\frac{1}{\varepsilon} A\left(\partial_{x}\right) U_{a}+\frac{1}{\varepsilon^{2}} A_{0} U_{a}=B\left(U_{a}, U_{a}\right)-\varepsilon^{2} R^{\varepsilon}, \\
U_{a}(0)=\left(\varepsilon \nabla^{T} \varphi_{0}, \psi_{0}, \varphi_{0}\right)^{T}+\varepsilon^{2} U_{2}(0)+\varepsilon^{3} U_{3}(0),
\end{array}\right.
$$

where

$$
\begin{aligned}
R^{\varepsilon}:= & 2 B\left(U_{0}, U_{2}\right)+B\left(U_{1}, U_{1}\right)+2 \varepsilon B\left(U_{1}, U_{2}\right)+\varepsilon^{2} B\left(U_{2}, U_{2}\right) \\
& -\sum_{n=2}^{3} \varepsilon^{n-2} \sum_{p} e^{-i p t / \varepsilon^{2}} \partial_{t} U_{n, p}-\sum_{p} e^{-i p t / \varepsilon^{2}} A\left(\partial_{x}\right) U_{3, p} .
\end{aligned}
$$

It is direct to check:

$$
\sup _{0<\varepsilon<1}\left(\left\|R^{\varepsilon}\right\|_{L^{\infty}\left(0, \infty ; H^{s-4}\right)}+\left\|U(0)-U_{a}(0)\right\|_{H^{s-4}}\right)<+\infty .
$$

Recall $s>d / 2+4$. Hence, by (7.34)-(7.38), this approximate solution $U_{a}$ fulfills Assumption 1.2 for the Cauchy problem (7.5)-(7.9).

\subsection{Partially strong transparency}

By (7.8) and (7.12), direct computation implies

$$
\Pi_{3}(\xi) B(\cdot, \cdot) \equiv 0
$$

By (7.11), we have

$$
R_{j, j^{\prime}, p}=\left\{\xi: \lambda_{j}(\xi)-\lambda_{j^{\prime}}(\xi)-p=0\right\}
$$


are all empty sets except

$$
R_{1,3,1}=\left\{\xi: \lambda_{1}(\xi)-1=0\right\}=\{0\}, \quad R_{2,3,-1}=\left\{\xi: \lambda_{2}(\xi)+1=0\right\}=\{0\} .
$$

Now we compute the interaction phases and the interaction coefficients corresponding to the non-empty resonance sets in (7.39). On one hand, direct computation gives

$$
\begin{aligned}
& \Pi_{1}(\xi) B\left(e_{1}\right) \Pi_{3}(\xi)=\frac{-\lambda}{2\left(d+|\xi|^{2}\right)}\left(\begin{array}{ccc}
\frac{i \xi \xi^{T}}{\lambda_{1}} & 0 & \frac{\xi|\xi|^{2}}{\lambda_{1}} \\
\frac{i \xi^{T}}{\lambda_{1}} & 0 & \frac{|\xi|^{2}}{\lambda_{1}} \\
\frac{-\xi^{T}}{\lambda_{1}} & 0 & \frac{i|\xi|^{2}}{\lambda_{1}}
\end{array}\right), \\
& \Pi_{2}(\xi) B\left(e_{-1}\right) \Pi_{3}(\xi)=\frac{-\lambda}{2\left(d+|\xi|^{2}\right)}\left(\begin{array}{cccc}
\frac{i \xi \xi^{T}}{\lambda_{2}} & 0 & \frac{\xi|\xi|^{2}}{\lambda_{2}} \\
\frac{i \xi^{T}}{\lambda_{2}} & 0 & \frac{|\xi|^{2}}{\lambda_{2}} \\
\frac{-\xi^{T}}{\lambda_{2}} & 0 & \frac{\left.i \xi\right|^{2}}{\lambda_{2}}
\end{array}\right)
\end{aligned}
$$

On the other hand, the interaction phases satisfy

$$
\left|\lambda_{1}(\xi)-1\right|^{-1}=\left|\lambda_{2}(\xi)-1\right|^{-1}=\frac{1}{\sqrt{1+|\xi|^{2}}-1}=\frac{\sqrt{1+|\xi|^{2}}+1}{|\xi|^{2}} .
$$

We find that $\left|\Pi_{1}(\xi) B\left(e_{1}\right) \Pi_{3}(\xi)\right| \cdot\left|\lambda_{1}(\xi)-1\right|^{-1}$ and $\left|\Pi_{2}(\xi) B\left(e_{-1}\right) \Pi_{3}(\xi)\right| \cdot \mid \lambda_{2}(\xi)+$ $\left.1\right|^{-1}$ are unbounded near resonance $\xi=0$. This implies that the strong transparency condition is not satisfied when $(i, j, p)=(1,3,1)$ or $(i, j, p)=(2,3,-1)$.

However, by (7.40) and (7.41), we can show that the following partially strong transparency condition is satisfied

$$
\left|\Pi_{1}(\xi) B\left(e_{1}\right) \Pi_{3}(\xi)\right| \leq C\left|\lambda_{1}(\xi)-1\right|^{1 / 2}, \quad\left|\Pi_{2}(\xi) B\left(e_{-1}\right) \Pi_{3}(\xi)\right| \leq C\left|\lambda_{2}(\xi)+1\right|^{1 / 2} .
$$

Thus, Assumption 1.6 is satisfied with $\alpha=1 / 2$. Moreover, the eigenvalue $\lambda_{3}$ is identically zero, which shows that Assumption 1.7 is also satisfied.

\subsection{Proof of Theorem 7.1}

All the assumptions introduced in Section 1.2 are verified for (7.5)-(7.9). By applying Theorem 1.8, we obtain

Theorem 7.2. There exists $\varepsilon_{0}>0$ such that for any $0<\varepsilon<\varepsilon_{0}$, the Cauchy problem (7.5)-(7.9) admits a unique solution $U \in L^{\infty}\left(0, \frac{T}{\varepsilon} ; H^{s-4}\right)$ for some $T>0$ independent of $\varepsilon$. Moreover, there holds

$$
\left\|U-U_{a}\right\|_{L^{\infty}\left(0, \frac{T}{\varepsilon} ; H^{s-4}\right)} \leq C \varepsilon,
$$

where $U_{a}$ is the approximate solution obtained in Section 7.4.2.

Theorem 7.1 is a direct corollary of Theorem 7.2. 


\section{Acknowledgements}

The authors thank Professor Weizhu Bao for helpful discussions. The first author acknowledges the support of the project LL1202 in the programme ERC-CZ funded by the Ministry of Education, Youth and Sports of the Czech Republic. Z. Zhang was partially supported by NSF of China under Grant 11371039 and 11425103.

\section{References}

[1] W. Bao, X. Dong, Analysis and comparison of numerical methods for the Klein-Gordon equation in the nonrelativistic limit regime, Numer. Math. 120 (2012), 189-229.

[2] C. Cheverry, O. Guès, G. Métivier, Oscillations fortes sur un champ linéairement dégénéré, Ann. Sci. Ecole Normale Sup. 36 (2003), 691-745.

[3] T. Colin, D. Lannes, Long-wave short-wave resonance for nonlinear geometric optics, Duke Math. J. 107 (2001), 351-419.

[4] T. Colin, D. Lannes, Justification of and long-wave correction to DaveyStewartson systems from quadratic hyperbolic systems, Discrete Contin. Dyn. Syst. 11 (2004), 83-100.

[5] T. Colin, G. Ebrard, G. Gallice, B. Texier, Justification of the Zakharov model from Klein-Gordon-waves systems, Comm. Partial Diff. Eq. 29 (2004), 13651401.

[6] P. Donnat, J.-L. Joly, G. Métivier, J. Rauch, Diffractive nonlinear geometric optics, Séminaire Equations aux Dérivées Partielles, Ecole Polytechnique, Palaiseau, 1995-1996, no. XVII, 25 pp.

[7] E. Dumas, About nonlinear geometric optics, Bol. Soc. Esp. Mat. Apl. SeMA No. 35 (2006) 7-41.

[8] J. Ginibre, G. Velo, The global Cauchy problem for the nonlinear Klein-Gordon equation, Math. Z. 189 (1985), 487-505.

[9] J. Ginibre, G. Velo, The global Cauchy problem for the nonlinear Klein-Gordon equation II, Ann. Inst. H. Poincaré Anal. Non Linéaire 6 (1989), 15-35.

[10] J.-L. Joly, G. Métivier, J. Rauch, Coherent and focusing multidimensional nonlinear geometric optics, Ann. Sci. Ecole Norm. Sup. 28 (1995), 51-113.

[11] J.-L. Joly, G. Métivier, J. Rauch, Diffractive nonlinear geometric optics with rectification, Indiana Univ. Math. J. 47 (1998), 1167-1241. 
[12] J.-L. Joly, G. Métivier, J. Rauch, Transparent nonlinear geometric optics and Maxwell-Bloch equations, J. Differential Equations 166 (2000), 175-250.

[13] S. Klainerman, Global existence of small amplitude solutions to nonlinear Klein-Gordon equations in four space-time dimensions, Comm. Pure Appl. Math. 38 (1985), 631-641.

[14] D. Lannes, J. Rauch, Validity of nonlinear geometric optics with times growing logarithmically, Proc. Amer. Math. Soc. 129 (2000), 1087-1096.

[15] P.D. Lax, Asymptotic solutions of oscillatory initial value problems, Duke Math. J. 24 (1957), 627-646.

[16] Y. Lu, High-frequency limit of the Maxwell-Landau-Lifshitz system in the diffractive optics regime, Asymptotic Anal. 82 (2013), 109-137.

[17] Y. Lu, Higher-order resonances and instability of high-frequency WKB solutions, J. Differential Equations 260 (2016), 2296-2353.

[18] Y. Lu, B. Texier, A stability criterion for high-frequency oscillations, Mém. Soc. Math. Fr. 142 (2015), vi+130pp.

[19] Y. Lu, Z. Zhang, Higher order asymptotic analysis of the nonlinear KleinGordon equation in the non-relativistic limit regime, preprint, 2015.

[20] A. Majda, Compressible fluid flows and systems of conservation laws in several space variables, Applied Math Sciences, Springer, vol. 53, 1984.

[21] N. Masmoudi, K. Nakanishi, From nonlinear Klein-Gordon equation to a system of coupled nonliner Schrödinger equations, Math. Ann. 324 (2002), 359-389.

[22] G. Métivier, Para-differential Calculus and Applications to the Cauchy Problem for Nonlinear Systems, Centro di Ricerca Matematica Ennio De Giorgi, CRM Series, vol. 5, Edizioni della Normale, Pisa, 2008, xii+140 pp.

[23] J. Shatah, Normal forms and quadratic nonlinear Klein-Gordon equations, Comm. Pure Appl. Math. 38 (1985), 685-696.

[24] B. Texier, The short wave limit for nonlinear, symmetric hyperbolic systems, Adv. Diff. Eq. 9 (2004), 1-52.

[25] B. Texier, Derivation of the Zakharov equations, Arch. Ration. Mech. Anal. 184 (2007), 121-183. 